UNITED STATES DEPARTMENT OF THE INTERIOR GEOLOGICAL SURVEY

HEWLETT PACKARD 9845 A COMPUTER PROGRAM TO INVERT COMPLEX RESISTIVITY DATA

\author{
James C. Washburne \\ Open-File Report 83-198 \\ 1983
}

This report is preliminary and has not been reviewed for conformity with the U.S. Geological Survey editorial standards.

Use of particular manufacturers and model numbers is for descriptive purposes only and does not constitute endorsement by the U.S. Geological Survey.

Although this program has been tested extensively, the U.S. Geological Survey cannot guarantee that it will give correct results in any or all particular applications. 
Introduction.............................

Program description

Input..............................

calculations...........................

output..............................

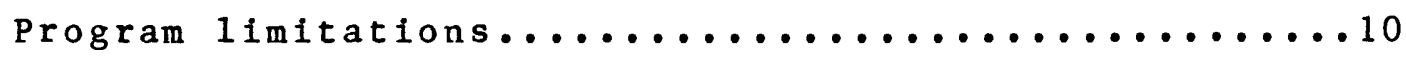

Procedural guidelines.......................11

General program conventions and notes.............12

User instructions: Program I/0................ 15

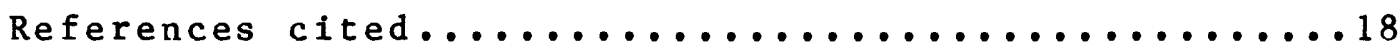

Figure 1a. Cole-Cole equivalent circuit model.

1b. A possible rock pore system.............

Figure 2. The effect of parametric variations

on a single Cole-Cole model............4

Figure 3. Cole-Cole model parameter correlations.....5

Table 1. List of symbols....................

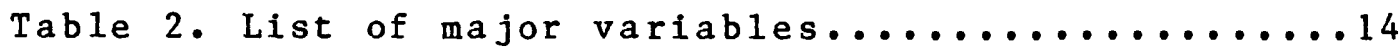

Appendix A. Example program 1isting..............19

Appendix B. Source program listing..............23 
Table 1--List of symbols

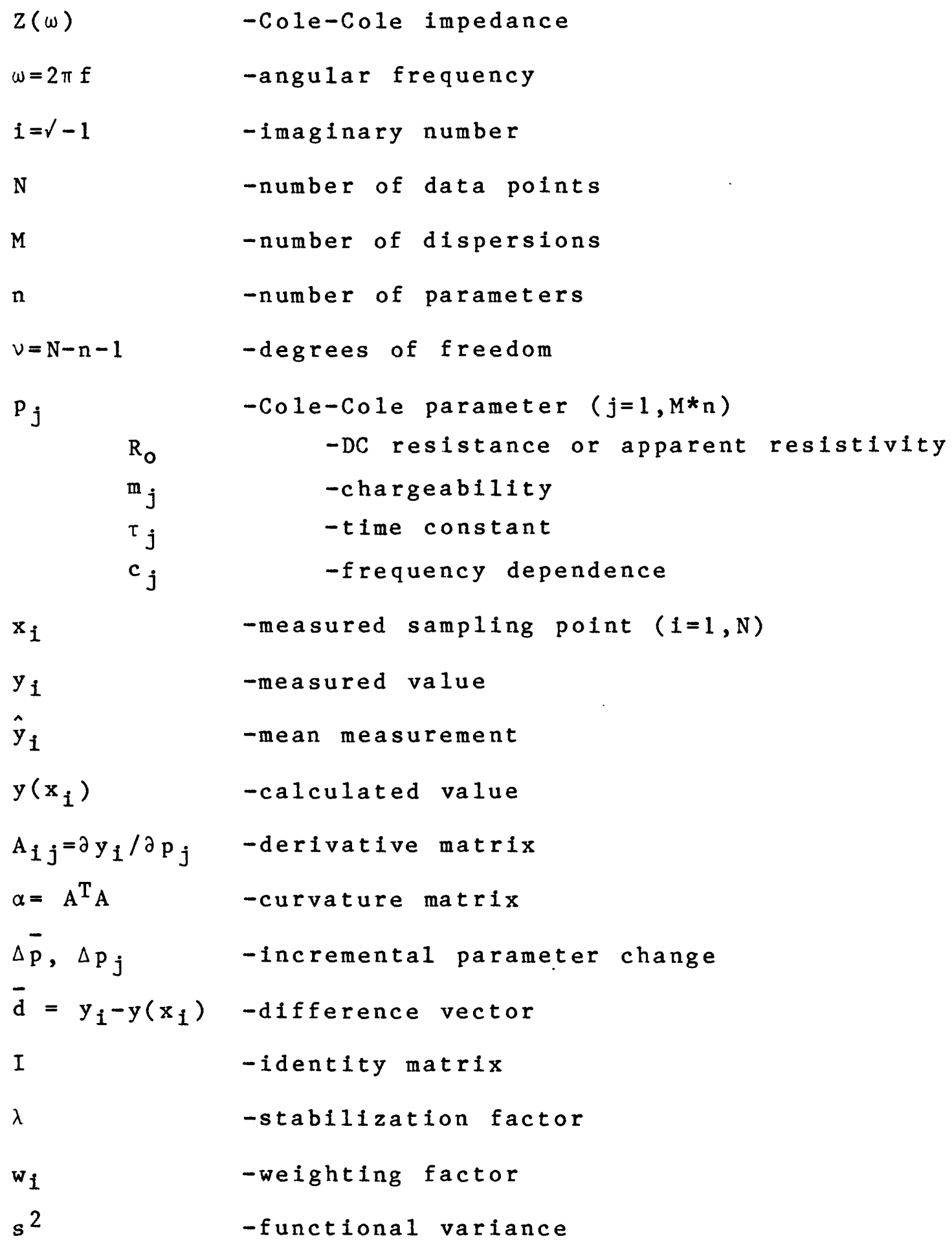




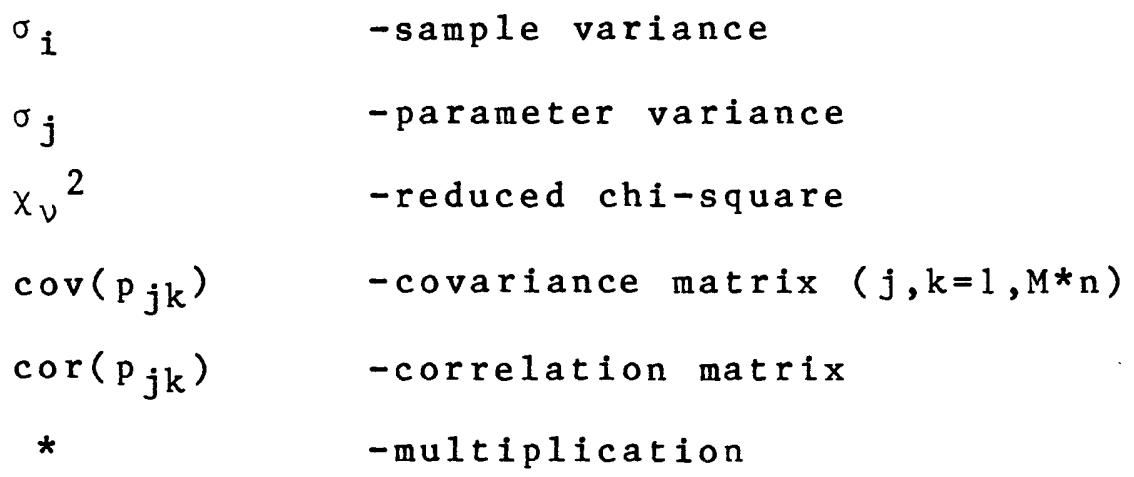


Introduction

This report describes an interactive Hewlett Packard (HP) $9845 \mathrm{~A}$ computer program that inverts spectral induced polarization (SIP) or complex resistivity (CR) data. A complex (that is, frequency dependent) impedance is defined in terms of a multiplicative Cole-Cole model (Cole and Cole, 1941; Pelton, 1977; Washburne, 1982). Either amplitude, phase or amplitude-phase data can be inverted. This program utilizes Marquardt's (1963) maximum neighborhood method to stabilize the inverted derivative matrix. The reduced chisquare (Bevington, 1969) determines convergence. Both 'normal' and reversed (or 'negative') dispersions can be modeled. This allows first-order EM coupling effects to be removed from field data. Program operation is demonstrated using a calculated dispersion.

Below, SIP measurements, Cole-Cole models and inversion techniques are introduced briefly. Relevant computational procedures are reviewed in three sections under "Program description": 1) input, 2) calculations and 3) output. After noting a few program limitations, user-orientated procedural guidelines, program notes and program input/output (I/O) are reviewed.

An SIP measurement consists of recording a complex impedance (usually resolved into amplitude-phase or realimaginary components) across a wide frequency range (.01$1000 \mathrm{~Hz}$, for instance). The objective of a SIP measurement is to quantify charge polarization effects that are induced in the earth by an alternating electric field. In most rocks, this polarization can be characterized by a simple dispersion or by several dispersions. Specific mechanisms or polarizable sources cannot be attributed to these dispersions, however, because a wide range of mechanisms and overlap of active zones exist among several possible sources. Until more of the physical processes governing natural rock polarization are understood, Cole-Cole parameterization is limited to simply quantifing the spectrum of a given measurement.

Cole-Cole parameters can be defined in terms of a simple equivalent circuit (fig. la) or related to a hypothetical rock pore system (fig. lb). More realistic situations consisting of a random network of these basic components also can be expressed in terms of a simple distributed circuit (Zonge, 1972). It is important to realize, however, that this solution is not unique in two 

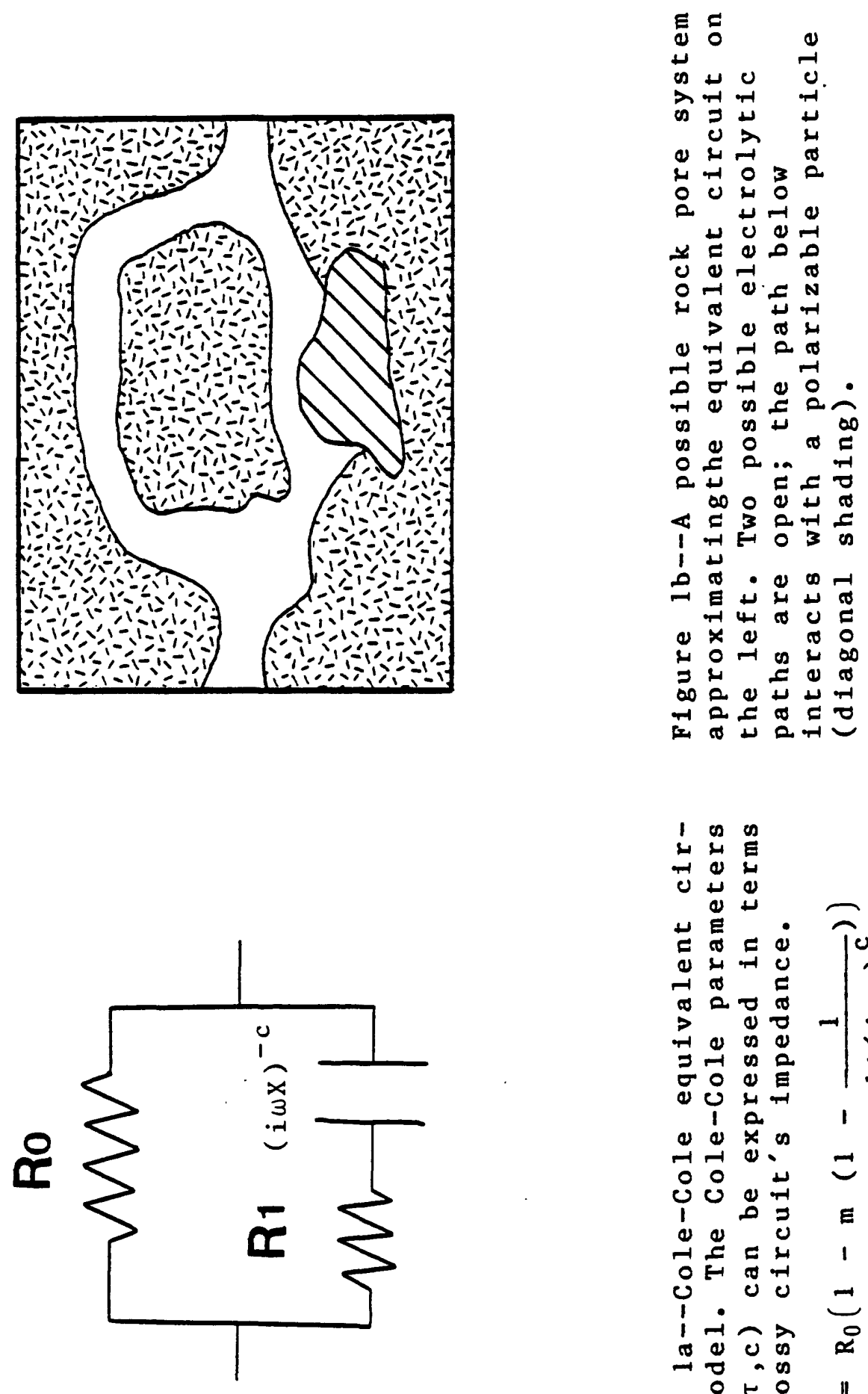

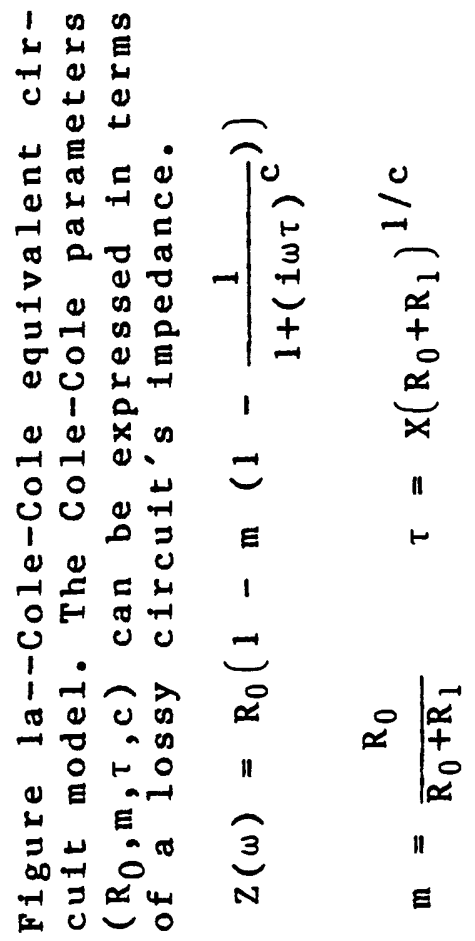


important respects: 1) the Cole-Cole model is only one of many possible mathematical representations and 2) inversion of Cole-Cole models involves a linearization of a nonlinear problem. Model parameters derived from noisy data are particularly nonunique. In general, an estimate of the parameters nonuniqueness can be obtained by carefully examining the inversion's output, which usually includes: 1) statistical measures, such as parameter standard deviation and correlation matrix and 2) intermediate parameterizations (large fluctuations are a sign of poor resolution).

Program description: Input

The program requires as input sets of frequency (Hz), amplitude (ohm-m) and phase (mrad) measurements in order of ascending frequency. Subroutine Test (1ine 6130) demonstrates how the data arrays are filled. Four methods of interactively weighting the data are possible (see "Program operation"). Amplitude data are scaled using a simple logarithmic transformation; phase data are scaled using a hyperbolic sine transformation. The second transformation scales small $(-1<x<1$ mrad) phases linearly while larger positive and negative phases are scaled logarithmically.

Initial Cole-Cole parameters (m, $\tau$ and $c$ ) must be supplied interactively. Several families of curves (fig. 2) demonstrate the effect each parameter has on a dispersion's shape. Any parameter can be held constant to constrain the final solution. A logarithmic transformation of $\tau$ effectively limits its range to between 4 and -7 . The ranges of $m$ and $c$ are physically constrained between 0 and 1 by the equivalent circuit representation of a Cole-Cole dispersion (Pelton etal., 1978). Constraining these two parameters with a simple trigonometric or hyperbolic transformation is ineffective because poorly resolvable parameters become fixed at unrealistic values. Therefore, it is best that these parameters be fixed as the need arises. Both $m$ and $c$ must be positive. Occasionally, a modified parameter becomes negative (it is displayed as a negative); however, its absolute value is used for subsequent calculations.

A weakness of the Cole-Cole model is the high inherent correlation between parameters. This is illustrated by inverting the systematically varying spectra in figure 2 and graphically summarizing the resulting parameter correlations (fig. 3). These results indicate that parametric ambiguity is greater when a dispersion is: strong (m), incomplete ( $\tau$ ) and/or broad (c). 


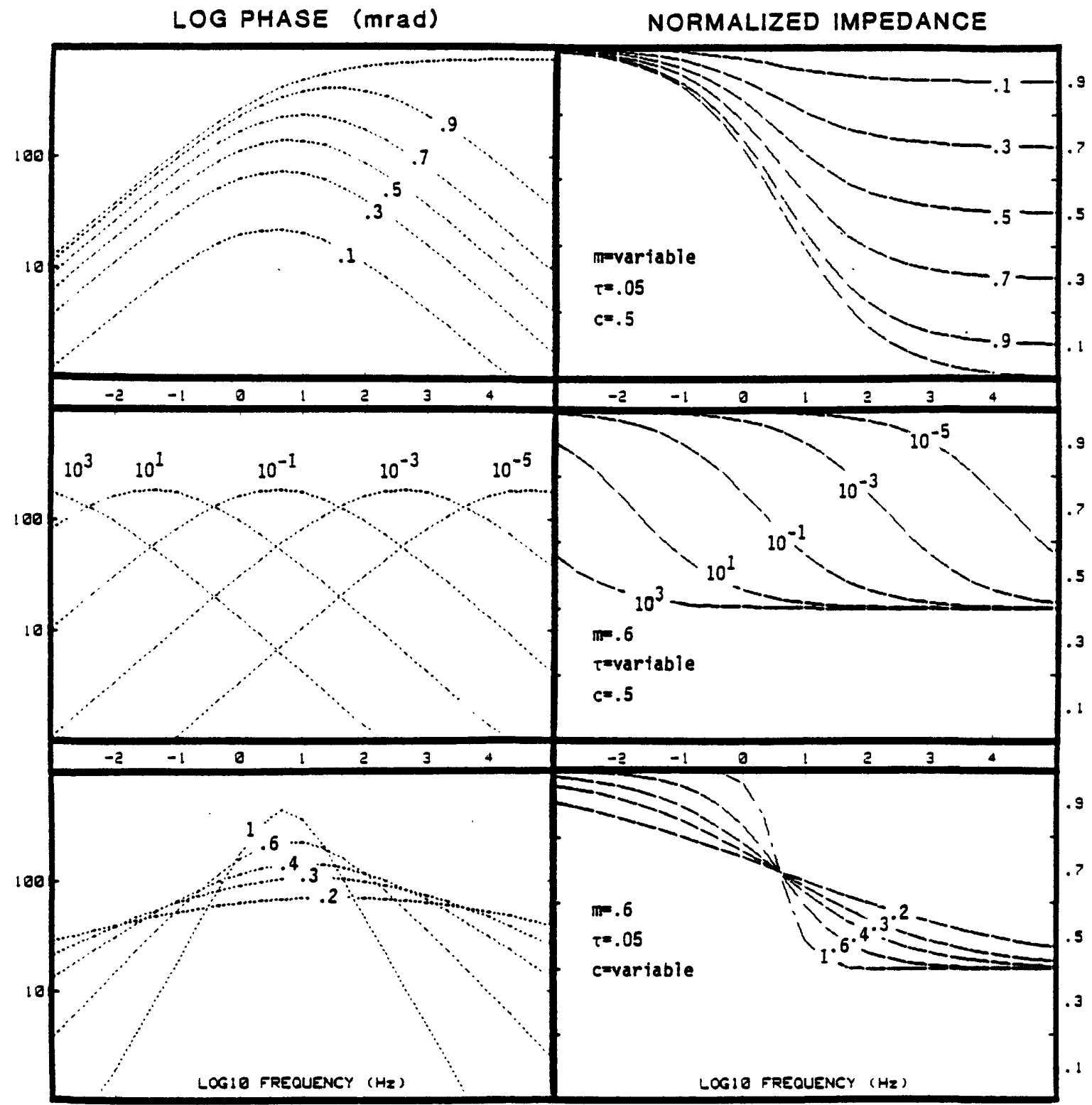

Figure 2--The effect of parametric variations on a single Cole-Cole model. Each family of curves was generated by holding two parameters constant and letting the third vary as indicated. 

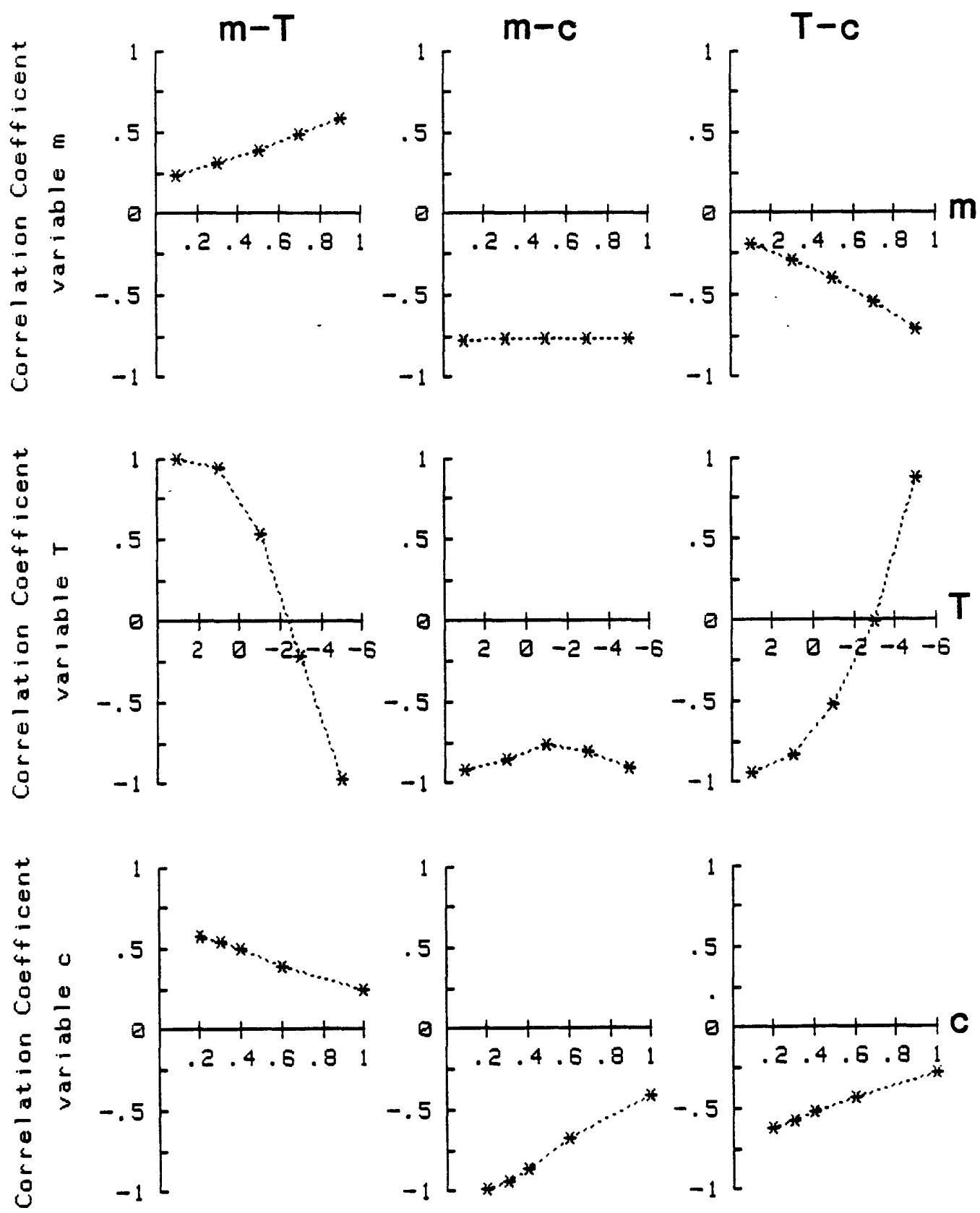

Figure 3--Cole-Cole model parameter correlations. Each dispersion in figure 2 has been inverted to form a measure of inherent parameter correlation. Constant parameters are $m, \tau, c=.6, .05, .5$. Variable parameters are plotted along the horizontal axis. 
Program description: Calculations

The forward solution (subroutine Colem) is expressed in terms of the real and imaginary parts of a complex impedance (equations 1-2), which is modeled by a product of Cole-cole dispersions (equation 3) (B.D. Smith, pers.commun., 1981; Washburne, 1982).

$$
\begin{aligned}
& \left.\mathrm{Z}_{\text {real }}=1-\frac{\mathrm{m}\left(a+(\omega \tau)^{2} \mathrm{c}\right.}{\mathrm{D}_{1}}\right) \\
& \mathrm{Z}_{\text {imag }}=-\frac{\mathrm{m} \mathrm{b}}{\mathrm{D}_{1}} \\
& \mathrm{Z}(\omega)=\mathrm{R}_{0}\left(1-\mathrm{m}_{1} \mathrm{f}_{1}\right)\left(1-\mathrm{m}_{2} \mathrm{f}_{2}\right)
\end{aligned}
$$

where $a=(\omega \tau)^{c} \cos (c \pi / 2)$

$$
\begin{aligned}
& \mathrm{b}=(\omega \tau)^{c} \sin (c \pi / 2) \\
& \mathrm{D}_{1}=1+2 a+(\omega \tau)^{2 c} \\
& \mathrm{f}=\left(1-\frac{1}{1+(i \omega \tau)^{c}}\right)
\end{aligned}
$$

(see table 1 for a definition of these and other variables). This parameterization is useful to quantify many spectral curves simply for more detailed interpretational analysis.

The inversion subroutine (Curfit) searches a nxn parameter space for a minimum reduced chi-square between the weighted raw and inverted data points (Bevington, 1969). The impedance function is linearized by considering only the first two terms in its Taylor-series expansion (equation 4), where $Z(\omega) / R_{0}$ has been transformed to $y(x)$ for simplicity.

$$
y_{i}=y\left(x_{i}\right)+\partial y_{i} / \partial p_{j} \Delta p_{j}
$$

In matrix notation this becomes (equation 5):

$$
\bar{d}=A \Delta p
$$

Multiplying each side by $A^{T}$ gives (equation 6):

$$
A^{T} \bar{d}=A^{T} A \Delta p
$$

A Marquardt-like stabilization factor ( $\lambda$ ) is added to the main diagonal of the curvature or normalized derivative 
A Marquardt-like stabilization factor $(\lambda)$ is added to the main diagonal of the curvature or normalized derivative matrix to keep it from becoming singular (Marquardt, 1963). This equation can now be solved for $\Delta p$ (equation 7 ).

$$
\Delta p=\left(A^{T} A+\lambda I\right)^{-1} A^{T} \bar{d}
$$

At each iteration, $\lambda$ is changed. Initially, and away from the zone of convergence, $\lambda$ is large and increasing-- the search approximating the gradient method. As a minimum is approached, however, $\lambda$ is decreased and the search approximates the linearized expansion method, which converges quadratically in this region. Convergence is reached when either the incremental or relative chi-square error becomes less than some tolerance. The tolerance defaults to $10^{-4}$ for phase and amplitude-phase inversion options and to $10^{-5}$ for the inversion of amplitude. These might need to be modified for exceedingly good or noisy data sets. Because these tolerances are derived from experience with theoretical, laboratory and field data sets, it appears as though the inversion of logarithmic amplitude data is less sensitive to parametric variations than is the inversion of logarithmic phase data.

Either numeric or analytic derivatives can be used in this program. The only significant difference between the two methods is the much greater speed at which analytic derivatives are calculated; the time savings is a function of the number and resolvability of the parameters. Numerical derivatives are calculated by perturbing each parameter, in turn, by $10 \%$ and finding the differential: $\Delta y / \Delta x$. Analytic derivatives are derived from the partial derivatives (equations 8-10) of a single Cole-Cole dispersion.

$$
\begin{aligned}
& \partial y / \partial m=1 / D_{1}\left[a+(\omega \tau)^{2 c}-i b\right] \\
& \partial y / \partial \tau=m c /\left(D_{1} \tau\right)[R+I] \\
& \partial y / \partial c=m / D_{1}^{2}[R \ln (\omega \tau)-I \pi / 2+i(R \pi / 2+I \ln (\omega \tau))] \\
& \text { where } R=2(\omega \tau)^{2 c}+a\left[1+(\omega \tau)^{2 c}\right] \\
& I=b\left[1-(\omega \tau)^{2 c}\right] .
\end{aligned}
$$

Since amplitude and phase are defined as (equations 11-12),

$$
A=\sqrt{Z_{\text {real }}^{2}+Z_{\text {imag }}^{2}}
$$




$$
\phi=\tan \left(Z_{\text {imag }} / Z_{\text {rea } 1}\right),
$$

analytic derivatives have the form of equations 13-14:

$$
\begin{array}{ll}
\frac{\partial A}{\partial p}=\frac{1}{A} & \left(\frac{\partial Z_{\text {real }}}{\partial p}+\frac{Z_{\text {imag }}}{Z_{\text {real }}} \frac{\partial Z_{\text {imag }}}{\partial p}\right) \\
\frac{\partial \phi}{\partial p}=\frac{1}{A^{2}} & \left(\frac{\partial Z_{\text {imag }}}{\partial p}-\frac{Z_{\text {imag }}}{Z_{\text {real }}} \frac{\partial Z_{\text {rea } 1}}{\partial p}\right)
\end{array} .
$$

Pelton etal. (1978) note that a Cole-Cole model whose frequency dependence is one can be used to decouple 'normal' high frequency EM coupling effects from lower frequency IP effects. Although parametric resolution is lost (Major and Silic, 1980) and this procedure is valid only before the phase maximum in simple geoelectric environments (Webster, 1980), it is a useful first approximation. 'Negative' coupling effects can be removed in a like manner by using two opposing Cole-Cole dispersions to model the coupling (Hallof and Pelton, 1980) (equations 15-16).

$$
\begin{aligned}
& \mathrm{Z}(\omega)=\mathrm{R}_{0}\left(1-\mathrm{m}_{1} \mathrm{f}_{1}\right)\left(1-\mathrm{m}_{2} \mathrm{f}_{2}\right) \\
& \mathrm{Z}(\omega)=\mathrm{R}_{0}\left(1-\mathrm{m}_{1} \mathrm{f}_{1}\right)\left(1-\mathrm{m}_{2} \mathrm{f}_{2}\right)\left(1+\mathrm{m}_{3} \mathrm{f}_{3}\right)
\end{aligned}
$$

Residual phases are calculated by decomposing the modeled real and imaginary parts of the spectrum from the real and imaginary (that is, cosine, sine) representation of the original spectrum.

Program description: Output

The program outputs: 1) inverted Cole-Cole parameters, 2) several statistical measures of the inversion's fit and the parameter's ambiguity and 3) a plot of the raw and inverted dispersions.

There are several measures of "goodness of fit". Bevington ( 1969 ) defines the functional variance ( $s^{2}$ ) of a set of data $\left(y_{i}\right)$ to a fitting function $\left(y\left(x_{i}\right)\right.$ ) as (equation 17):

$$
s^{2}=1 / \nu \sum_{i} w_{i}\left[y_{i}-y\left(x_{i}\right)\right]^{2}
$$

where the weighting function, $w_{i}$ (equation 18), is normalized in terms of the sample variances, $\sigma_{i}$ (equation 19). 


$$
\begin{aligned}
& w_{i}=\frac{1 / \sigma_{i}^{2}}{1 / N \sum_{i} 1 / \sigma_{i}^{2}} \\
& \sigma_{i}=\frac{1}{N} \sum_{i}\left(y_{i}-\hat{y}_{i}\right)^{2}
\end{aligned}
$$

The reduced chi-square $\left(x_{\nu}^{2}\right)$ (equation 20) is a measure of how well a fitting function matches a series of observations and is found by taking the ratio of the functional yariance to the weighted average of the sample variances $\left(\sigma_{i}^{2}=w_{i} \sigma_{i}^{2}\right)$.

$$
x_{\nu}^{2}=1 / \nu \quad \sum_{i}\left[y_{i}-y\left(x_{i}\right)\right]^{2}=s^{2} / \bar{\sigma}_{i}^{2}
$$

Ideally, the reduced chi-square is unity-- larger values indicating a poor fit, smaller values indicating a larger uncertainty, in the observations. If sample variance is unknown, $\sigma_{i} 2=1$, and the reduced chi-square is simply the least-squares error.

Parameter error can be estimated from the covariance matrix (equation 21).

$$
\operatorname{cov}\left(p_{j k}\right)=\left[A^{T_{A}}\right]^{-1} x_{\nu}^{2}
$$

Diagonal elements of $\operatorname{cov}\left(p_{j k}\right)$ indicate parameter variance $\left(\sigma_{j}\right)$, non-diagonal elements indicate parameter covariance. For reasonable parameterizations, the normalized covariance or correlation matrix (equation 22) gives a measure of the correlation between parameters.

$$
\operatorname{cor}\left(p_{j k}\right)=\frac{\operatorname{cov} p_{j k}}{\sqrt{\operatorname{cov} p_{j j} \operatorname{cov} p_{k k}}}
$$

A correlation of zero indicates an independent parameter. Coefficients of +1 and -1 indicate a strong correlation or inverse correlation, respectively.

Following the statistics, raw and inverted dispersions are plotted. A plot of logarithmic phase lag and normalized (by $R_{0}$ ) amplitude versus logarithmic frequency is preferred because Cole-Cole parameters are estimated easily from this presentation. Data can be plotted on a normalized realimaginary axis diagram (also known as an Argand or Cole-Cole diagram). For the benefit of those who prefer this format, the raw data are plotted on a reduced real-imaginary diagram also. 
Program limitations

When comparing this data to other Cole-Cole modeled data, beware of slight but parametrically significant differences in the way multiple dispersions are defined.

Three dispersion models are adequate to fit most laboratory and field SIP data. If more complicated models are desired, the $9845 \mathrm{~A}^{\prime} \mathrm{s} 64 \mathrm{~K}$ byte memory must be extended or advantage taken of overlapped processing.

The validity of inversion statistics depends upon: 1) spectral resolvability in terms of the Cole-Cole model and 2) linearity of the Taylor-series approximation. At best, statistics serve only as relative indications of parametric stability and correlation.

The Cole-Cole model only approximates EM coupling. As a result, parameters associated with high frequency polarization dispersions are ambiguous. In general, however, this is a good enough approximation so that parameters associated with low frequency dispersions are more characteristic of a coupling-free environment. 


\section{Procedural Guidelines}

1) Weight to 0 those points that are not part of a smooth spectral curve. One 'bad' point, especially at a frequency extreme, can wreck convergence.

2) Determine how many polarization dispersions are present. Be sure to note models that do not truly correspond to a single dispersion (that is, where impulsive or transitory behavior is not fit well by a simpler model). Minimize the number of models.

3) Initialize the model. Hold unresolvable parameters constant. Refer to Figure 2 if you have difficulty relating spectral shape to the cole-Cole parameters. In general, resolvable parameters are characterized by: 1) clear non-zero slopes ( $>4$ pts) and 2) clearly distinguishable phase peaks ( $>50$ mrad); unresolvable parameters are characterized by: 1) very low slopes and phase peaks (m, $\tau, c)$ and 2) phase maxima outside the measuring range $(m, \tau)$. First, minimize the number of constant parameters. Later, use insight gained from a set of spectra to determine which of the most ambiguous parameters to hold constant.

4) There will always be a better fitting model. When the data is fit adequately, stop inverting and start interpreting! 
General Program Conventions and Notes

SYSTEM: Hewlett Packard (HP) 9845A/ 64K byte memory. Refer questions regarding HP BASIC syntax to the HP 9845 manuals: "Operating and programing" and "Graphics programing techniques".

UNITS: Unless otherwise noted, assume the following units: meters, milliradians, ohm-meters, volts, amperes.

DEFAULTS: All questions have default values preasigned. Pushing CONT (continue) executes these defaults. The default response is indicated by underlining.

VARIABLES: Variable names are usually mnemonics related to their function. In general, a given variable's definition is ubiquitous to the whole program. This program's most important variables are defined in table 2 .

PROGRAM HEADERS: Definitions of parameters passed to each subroutine are found in its heading. Array dimensions are indicated in parenthesis. Descriptive line labels provide access to the head of each subroutine if this is required.

USER MODIFICATIONS: There are a few places where the user might want to set a default variable in the program. A few of the most likely modifications are described below.

Begin by pushing EDITLINE ...; finish by pushing STORE.

1) The internal printer is activated by changing the device code, Pdev, in line 390 from 16 to 0 . Activating lines 2100 and 2250 will keep intermediate parameters from being printed out when Pdev=0.

2) A double logarithmic phase scale (to plot both positive and negative phases) is activated by changing the variable Log\$ in line 400 from "YES" to "DBL".

3) Numeric, rather than analytic derivatives, will be calculated if the variable Analytics in line 1080 is changed from "YES" to "NO".

4) The default input device variable Devices can be changed in line 6240 from ":C" (disk) to ":T14" (1eft-hand tape drive), for instance. 
5) The log-log plot's base and frequency range are easily modified in lines 750 and 5300. These default to: base $=10, f_{m i n}=-3$ and $f_{\text {max }}=5$. Either plot can be suppressed by deactivating (with F! $_{\text {) }}$ ) the appropriate line (see lines $750,770,5300$ or 5310). Note that if subroutine Crplt is not called before Argplt, the plotter must be initialized. Change line 8910 to: PLOTTER IS 13, "GRAPHICS"-- to accomplish this.

6) Occasionally, weak dispersions are incorrectly scaled in the final plot. This condition exists when a large DC offset is obvious between the raw and inverted normalized amplitudes. To replot the data correctly, temporarily modify $\mathrm{Rk}$ in line 7070 from 1 to RO. 
Table 2--List of major variables

Below, the most important common, passed and calculated variables or arrays are defined. Default values are indicated in parentheses.

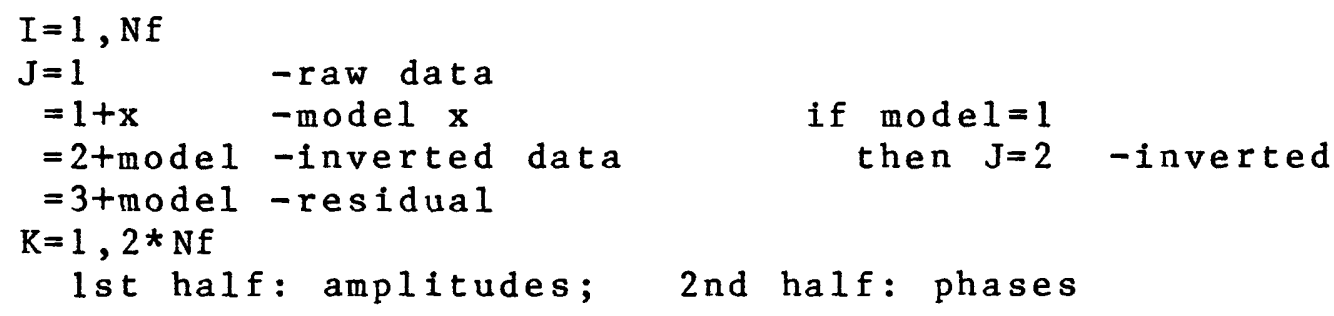

COMMON:

Nf -number of frequencies

model -number of Cole-Cole dispersions modeled

PASSED :

$$
\begin{array}{ll}
Y(1, K) & \text {-input or raw data } \\
\text { Yfit }(1, K) & \text {-output or best fit } \\
\text { Sigy }(1, K) & \text {-standard deviations } \\
\text { Ywt }(1, K) & \text {-data weights } \\
P(3, \operatorname{mode} 1) & \text { Cole-Cole parameters }
\end{array}
$$

CALCULATED :

$$
\begin{array}{ll}
\mathrm{A}(\mathrm{I}, \operatorname{mode} 1 * 3) & \text { Derivative matrix } \\
\mathrm{Dp}(\operatorname{mode} 1 * 3, \operatorname{mode} 1 * 3) & \text { Inverted incremental parameters }
\end{array}
$$


User Instructions: Program I/O

The following example uses the theoreticallycalculated response of two RC circuits in series. Both circuits have a Warburg-like ( $\sqrt{ } f)$ capacitance. Below, a typical program run (Iisted in appendix A) is broken-down into 16 steps. System commands are capitalized. Commonly occurring program variables are offset by a double hyphen $(--X)$.

A. Turn on the machine and insert the program tape into the right-hand $(\mathrm{T}-15)$ tape drive. Insert the data tape into the left-hand $(T-14)$ tape drive.

B. Type GET "CRDBL", and press EXECUTE.

C. When the program is loaded, press RUN. Several prompting statements will appear successively on the screen and the user should type the appropriate responses as follows:

1) Today's date? (Mo/Da/Yr) End by pressing CoNT. --Date\$

2) Read from? : --Iofile $\$$ Choose the proper subroutine to read in the data, CONT.

3) File name? : Enter the data file name, ConT. --Fnm\$

4) Do you want to PLOT or ... CONT? : This allows the data to be previewed for the purpose of weighting points and choosing the initial inversion model. After generating the plot, the program waits three seconds and will continue automatically unless PAUSE is executed.

5) What do you want to invert? Type one number, CONT. --Iop

6) Choose one method of weighting the data? Four modes are possible:

Mode 1: Instrumenta1- use when measurement standard deviations are known.

Mode 2: Statistical-assumes a statistical relationship where errors are proportional to magnitude.

Mode 3: Selective- allows arbitrary weights to be assigned to each data point. In turn, amplitude and phase data are displayed, five points per line. A row of "l"'s, also offset in groups of five, will be displayed at the bottom of the screen. These are default weights. Use the $(--$ and $-\rightarrow$ keys to position the cursor under the data 
point that is to be reweighted. CONT when finished.

Mode 4: None- assigns equal weights of "1" to each data point (default).

7) At this point, a tabulated summary of the data is printed to the device specified by Pdev (line 390). In this example, frequency ( $\mathrm{Hz}$ ), amplitude (ohm-m) and phase (mrad) are printed with their respective weights. Note that these are values of phase shift whereas phase lag is plotted.

8) How many dispersions ... do you want to model? --model Refer to "Procedures". End with CONT.

9) Input $\mathrm{M}, \mathrm{T}, \mathrm{C}$ :

Requests initial Cole-Cole parameters, CONT.

10) Are these correct?: Requests confirmation that parameters have been entered correctly. Typing No branches back to step 9. CONT.

11) Do you want to hold one parameter constant?: Allows one parameter of current model to be held invariant during inversion. CONT.

12) "Which one?:" Requests parameter to be held constant. This loop must be passed through for each parameter held constant. CONT.

At this point, the computer starts inverting the data. If, for any reason, the inversion is terminated prematurely by pushing special function key "k8" (ie. wildly fluctuating or unrealistic parameters), the program should jump to step 13 (ie, statistics will be calculated for the last iteration). Unfortunately, if $k 8$ is pushed during an intermediate calculation, the statistics and inverted plot might be invalid. Avoid this situation by pushing $k$ immediately following the display of an intermediate result.

13) Intermediate results are printed on the device specified by Pdev following each iteration. This output consists of:

a) Fixed parameters, if any.

b) Free Cole-Cole parameters for each iteration. The column headings are:

It: iteration -iteration 0 lists initial parameters. Lamda( $\lambda)$ : Marquardt stabilization factor. 
Rchisqr: Reduced chi-square.

$M, T, C:$ Cole-Cole parameters $m, \tau$ and $c$.

c) \% Sigma= parameter standard deviations.

d) Correlation matrix. If inversion option (Iop) 1 or 3 was chosen (amplitude or both, respectively), an additional parameter ( DC resistivity) is used to find parameter correlations. Its relationship to other parameters is found in the matrix's last row.

14) Type YES to plot your results. Type No to change parameters, CONT. Program loops back to step 8 if this parameterization is not satisfactory. For the novice using this program, 'bad' data points are the cause of poor parameterizations more often than are unresolvable dispersions. If this is the case, start over from the beginning by pushing STOP twice and then RUN.

15) Which dispersion do you want to remove? Enter the dispersion or model number, CONT. This step is skipped if 0 is typed.

16) The final plot is automatically displayed on the screen. Type DUMP GRAPHICS and press EXECUTE, to get a hard copy.

WHAT IS PLOTTED:

Normalized amplitude $\left(Z / R_{0}\right)$

(0)

Inverted amplitude

$\log _{10}$ phase lag

Inverted dispersions

Residual phase

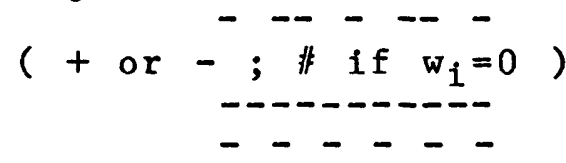

$R_{\mathrm{dc}}=\mathrm{R}_{\mathrm{o}}$ : Calculated zero frequency apparent resistivity

A real-imaginary axis diagram of the data is made in the lower left-hand corner. Each curve is normalized by $R_{0}$ • The axes are negative upwards and to the left and are graduated at $10 \%$ increments and labeled every. 5 units. Only the inverted dispersion is plotted (see USER MODIFICATIONS). Points weighted to zero are skipped. Plus (t) symbols mark every fourth frequency point. 


\section{RE FE RE NCES CITED}

Bevington, P.H., 1969 , Data reduction and error analysis for the physical sciences: New York, McGraw-Hill, 330 p.

Cole, K.S. and Cole, R.H., 1941, Dispersion and absorption in dielectrics: Journal of Chemical Physics, V.9, p. 341.

Hallof, P.G. and Pelton, W.H., 1980, The removal of inductive coupling effects from spectral IP data: Paper presented at the 50 th Annual SEG International Meeting, Houston, Nov. $16-20,25 \mathrm{p}$.

Major, J. and Silic, J., 1981, Restrictions on the use of Cole-Cole dispersion models in complex resistivity interpretation: Geophysics, V.46(6), p. 916-931.

Marquardt, D.W., 1963, An algorithm for least-squares estimation of nonlinear parameters: Journal of Industrial and Applied Mathamatics, V.2(2), p. 431-441.

Pelton, W.H., 1977 , Interpretation of induced polarization and resistivity data: Unive of Utah, Ph. D. Thesis, 255 p.

Pelton, W.H., Ward, S.H., Hallof, P.G., Sill, W.R., and Nelson, P.H., 1978 , Mineral discrimination and removal of inductive coupling with multifrequency I.P.: Geophysics, V.43(3), p. 588-609.

Washburne, J.C., 1982, Parameterization of spectral induced polarization data and in situ and laboratory spectral induced polarization measurements: West Shasta copper-zinc district, Shasta, CA.: Colorado School of Mines, M.S. Thesis, 443 p.

Webster, S.S., 1980, Implications of a spectral IP survey at Elura, in D.W. Emerson, ed., The geophysics of the Elura orebody, Cobar, New South Wales: Bulletin of the Australian Society of Exploration Geophysicists, V.11(4), p. 201-207.

Zonge, K.L., 1972, Electrical properties of rocks as applied to geophysical prospecting: University of Arizona, $\mathrm{Ph}$. D. thesis, 153 p. 


\section{Appendix A \\ Example Program Listing}

The following listing was made by assigning PRINT ALL IS 0 and depressing the PRINT ALL key. Each step is described in the User Instructions section of this report. Under normal operating conditions, only the two output sections indicated by a double line in the right-hand margin will be output to the thermal printer when Pdev=0. 
1) Enter todays date(m/d/y):

$1 / 31 / 82$

2) Read data from TEST, In 1, or In2?

2) TEST

3) TEST:F i lename?

3) $m R C \operatorname{ser}$

4) Do you warit to PLOT and prewiew the spectrum or CONT? PLOT

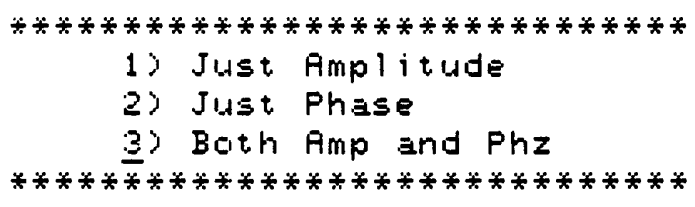

2) Just Phase

3) Both Amp and Phz

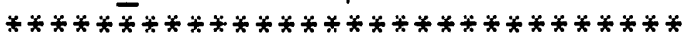

5) What do you want to invert?

3

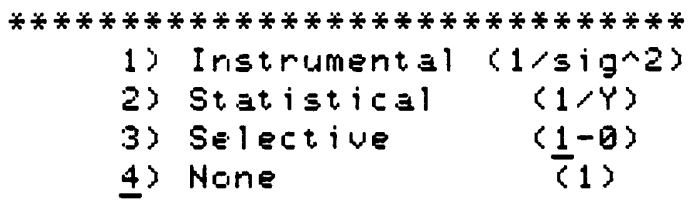

6) Choose one method of weighting the dat a? 3

AMPL I TUDE

\begin{tabular}{|c|c|c|c|c|}
\hline $\begin{array}{l}1.97 E+010 \\
1.68 E+00 \\
1.35 E+00 \\
1.04 E+00\end{array}$ & $\begin{array}{l}1.95 E+00 \\
1.60 E+00 \\
1.28 E+00 \\
1.02 E+00\end{array}$ & $\begin{array}{l}1.91 E+50 \\
1.54 E+00 \\
1.19 E+00\end{array}$ & $\begin{array}{l}1.85 E+\square D \\
1.49 E+0 \theta \\
1.12 E+0 \theta\end{array}$ & $\begin{array}{l}1.77 E+0 \square \\
1.43 E+0 \square \\
1.97 E+0 \theta\end{array}$ \\
\hline
\end{tabular}

PHASE

$\begin{array}{lllll}-1.41 E+01 & -2.31 E+91 & -3.53 E+01 & -5.01 E+01 & -6.14 E+01 \\ -5.41 E+01 & -5.91 E+01 & -5.33 E+01 & -5.35 E+01 & -6.18 E+01 \\ -7.54 E+01 & -8.63 E+01 & -8.51 E+01 & -7.08 E+01 & -5.10 E+01 \\ -3.32 E+01 & -2.03 E+01 & & & \end{array}$

Edit the weighting function; then CONT.

$1,1,1,1,1|1,0,1,1,1| 1,1,1,1,1 \mid 1,1$ 
7) FREQUENCY

$3.16 \mathrm{E}-93$

1. $00 E-02$

$3.16 E-02$

1. 00E-01

3. $16 E-01$

1. $00 E+00$

3. $16 E+00$

1. $.0 E E+\square 1$

3. $16 E+01$

1. $00 E+02$

3. $16 E+02$

1. $0 \mathrm{DE}+03$

3. $16 E+03$

1. $00 E+04$

3. $16 E+04$

1. $00 E+05$

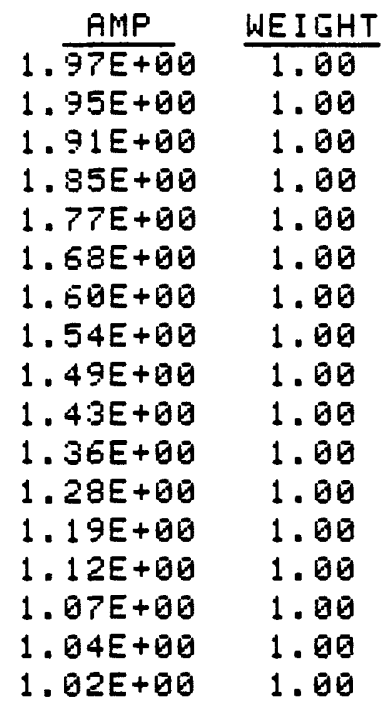

$\frac{P H Z}{41 E+\overline{1} 1}$
$-2.31 E+\square 1$

WE IGHT

$-3.57 E+01$

$-5.01 E+01$

$-6.14 E+\theta 1$

$-6.41 E+01$

$-5.91 E+01$

$-5.33 E+01$

$-5.35 E+01$

$-6.18 E+01$

$-7.54 E+01$

$-8.63 E+01$

$-8.51 E+01$

$-7.08 E+01$

$-5.10 E+01$

$-3.32 E+\square 1$

1.00

1.00

1.00

1.00

1.00

0.00

1.00

1.00

1.00

1.00

1.00

1.00

1.00

1.00

1.00

$-2.03 E+01$

1.00

8) How many dispersions?(default:2, max:3)

\section{2}

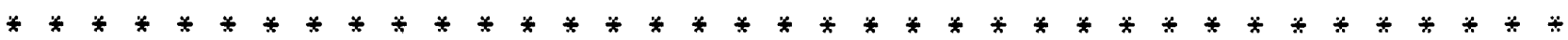
MODEL

9) INPUT $M, T, C:$

$.5,1, .5$

CHARIEABILITY $=.500$ TIME CONSTANT $=1$. DOE+00 FREQUENCY'DEPENDEHCE $=.500$

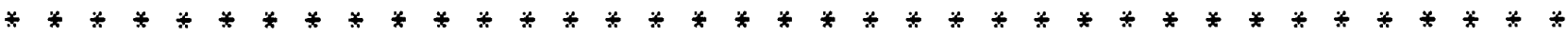

10) Are these correct?(YES or NO)

YES

11) Do you warit to hold one parameter constant?(YES or No)

YES

12) 3

Enter one parameter you want to hold constant $(1,2$, or 3$)$ :

Do you warit. to hold one parameter constant? (YES or NO)

NO

MODEL

2

INPUT $M, T, C:$

$.5, .001, .3$

CHARLEABILITY $=.500$ TIME COHSTANT $=1$. DOE-13 F FREQUENC' DEPENDENCE $=.300$

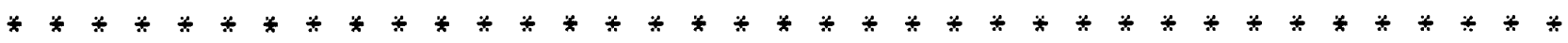

Are these correct? YYES or NO〉

YES

Do you warit to hold one parameter constant? (YES or NO)

NO

Don't bother me, I'm computing. . Pressks to terminate inversion 
13) CONSTANT PARAMETERS: C1: 5.00E-91

\begin{tabular}{|c|c|c|c|c|c|c|}
\hline 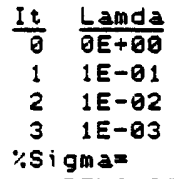 & $\begin{array}{l}\frac{\operatorname{Rchisg}}{3.96 E-\theta 1} \\
1.93 E-02 \\
2.52 E-04 \\
4.41 E-08\end{array}$ & $\begin{array}{l}\text { 5. } \frac{M 1}{0 \theta E-\theta 1} \\
\text { 2. } 25 E-\theta 1 \\
2.5 \theta E-\theta 1 \\
2.48 E-91 \\
3.86 E-02\end{array}$ & $\begin{array}{l}\text { 1. } \frac{T 1}{0 \theta E}+\theta \theta \\
9.55 E-\theta 1 \\
1.24 E+\theta \theta \\
1.2 \theta E+\theta \theta \\
3.19 E-\theta 2\end{array}$ & 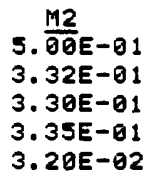 & $\begin{array}{l}\text { 1. } \frac{T 2}{10 E-03} \\
5.99 E-04 \\
3.69 E-04 \\
4.00 E-04 \\
3.77 E-02\end{array}$ & $\begin{array}{l}\text { 3. } \frac{C 2}{0 \theta E-01} \\
4.35 E-01 \\
4.99 E-01 \\
5.00 E-01 \\
2.92 E-02\end{array}$ \\
\hline
\end{tabular}

CORRELATION MATRIX

$\begin{array}{rrrr}1 & 1.000 & & \\ 2 & -.732 & 1.000 & \\ 3 & -.650 & .588 & 1.000 \\ 4 & -.710 & .616 & .714 \\ 5 & .587 & -.451 & -.588 \\ 6 & .219 & -.071 & .038\end{array}$

$\begin{array}{rr}1.000 & \\ -.677 & 1.000 \\ -.009 & .057\end{array}$

The reduced chi square has converged or diverged.

Type YES to plot your results. Type No to change parameters.

14) PLOT?

YES

15) Which dispersion do you want to remove $(\underline{\theta}, 1,2,3)$ ?

16) DUMP IRAPHICS

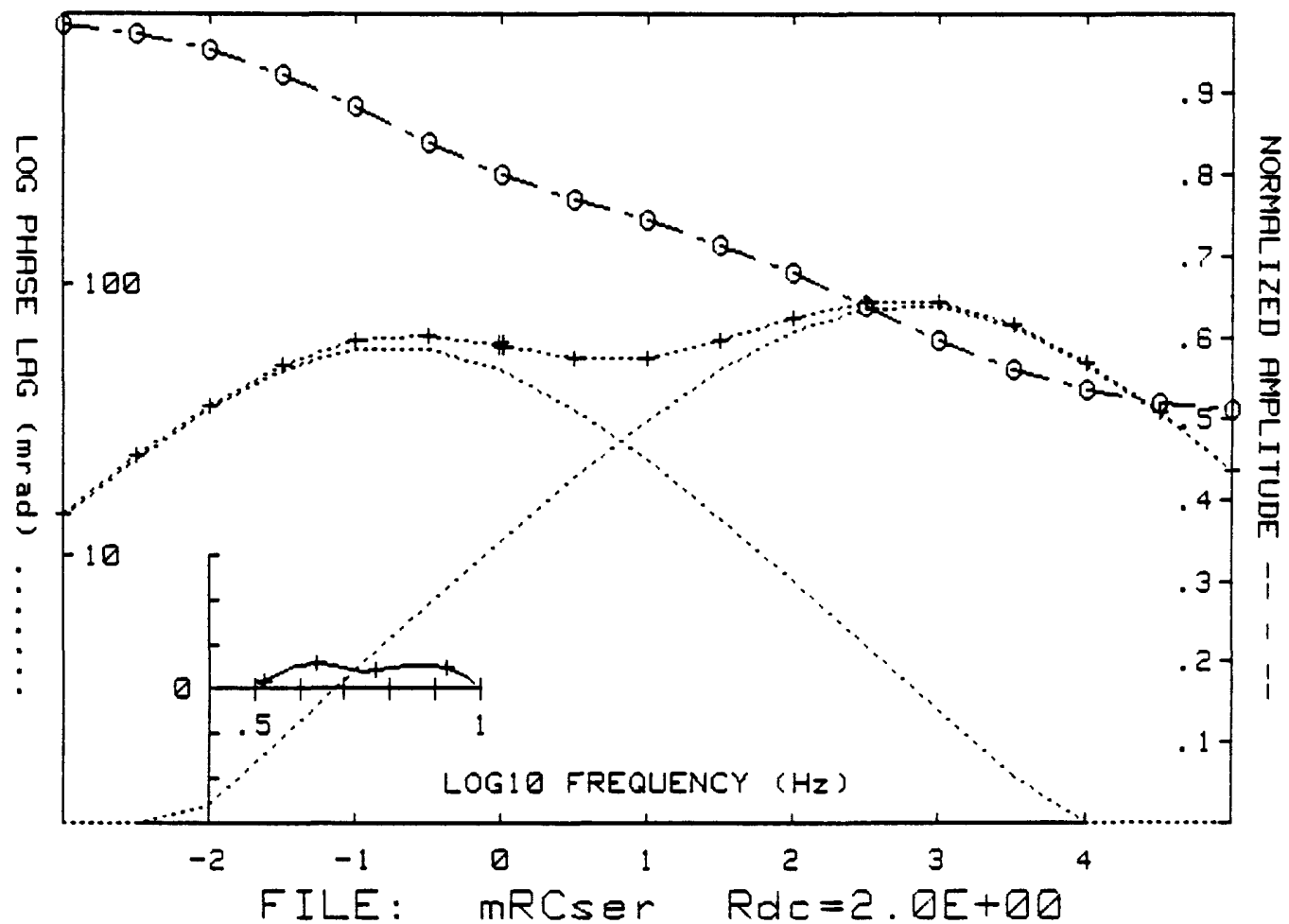


Appendix B

Source Program Listing 


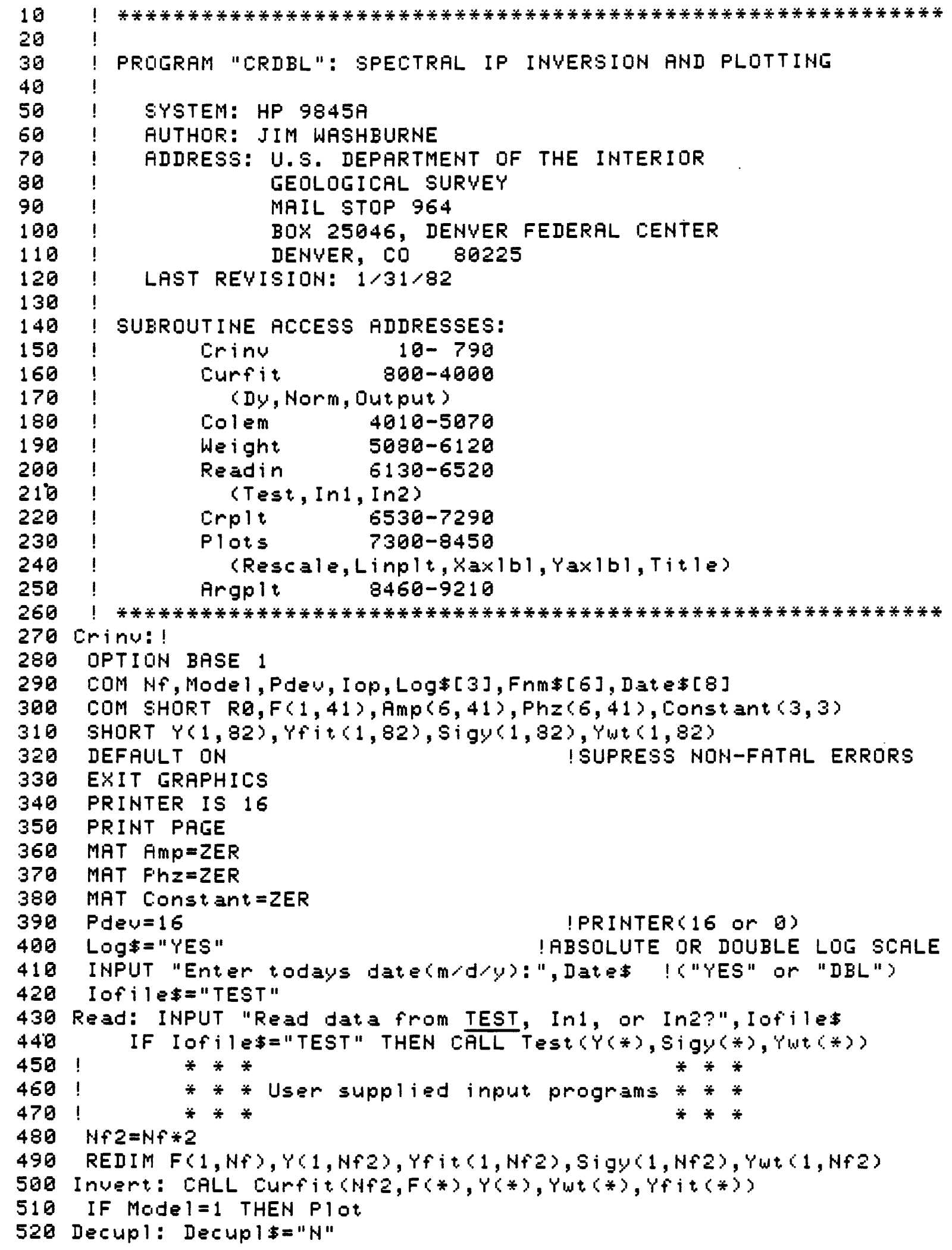


$530 \quad$ Ans $=0$

540 INPUT "Which dispersion do you want to remove(g, 1, 2, 3)?", Ans

550 IF ARIS=0 THEN Plot

560 Decupl $\$=" Y "$

$570 \quad M=$ Ans +1

$580 \mathrm{RK}=1$

590 IF $(F m p(M, 1)>1.5\rangle$ OR $(A m p(M, 1)<.5\rangle$ THEN RK=RO

600 Last $=$ Mode $1+3$

610 FOR I $=1$ TO $\mathrm{NF}$

$620 \quad R 0=A m p(1, I) / R \theta * \operatorname{CoS}(P h z(1, I) / 1000)$

$630 I \quad I 0=A m p(1, I) / R 0 * S I N(P h z(1, I) / 1000)$

640 Re $=A m p(M, I) / R k * \operatorname{CoS}(P h z(M, I) / 1000)$

650 Im $I m p(M, I\rangle) R K * S I N\langle P h z\langle M, I\rangle) 1000\rangle$

$660 \quad R 2=1-R E$

$670 \quad I 2=-I \mathrm{~m}$

$680 \quad D r=I 2 \wedge 2+(1-R 2) \wedge 2$

$690 \quad R r=\langle R o *(1-R 2)-I 0 * I 2)>D r$

$700 \quad I r=(R O * I 2+I 0 *(1-R 2)\rangle)>D r$

$710 \quad A m p(L a s t, I)=R 0 * S Q R(R r \wedge 2+I r \wedge 2\rangle$

$720 \quad P H_{1} z(L a s t, I)=1000 * A T H(I r / R r)$

730 NEXT I

740 !

750 Plot: CALL Crplt $(10,-3,5$, Decupl\$, Ywt (*)

$760 \quad 1$

770 CALL Frgplt (Ywt:

780 STOP

790 END 


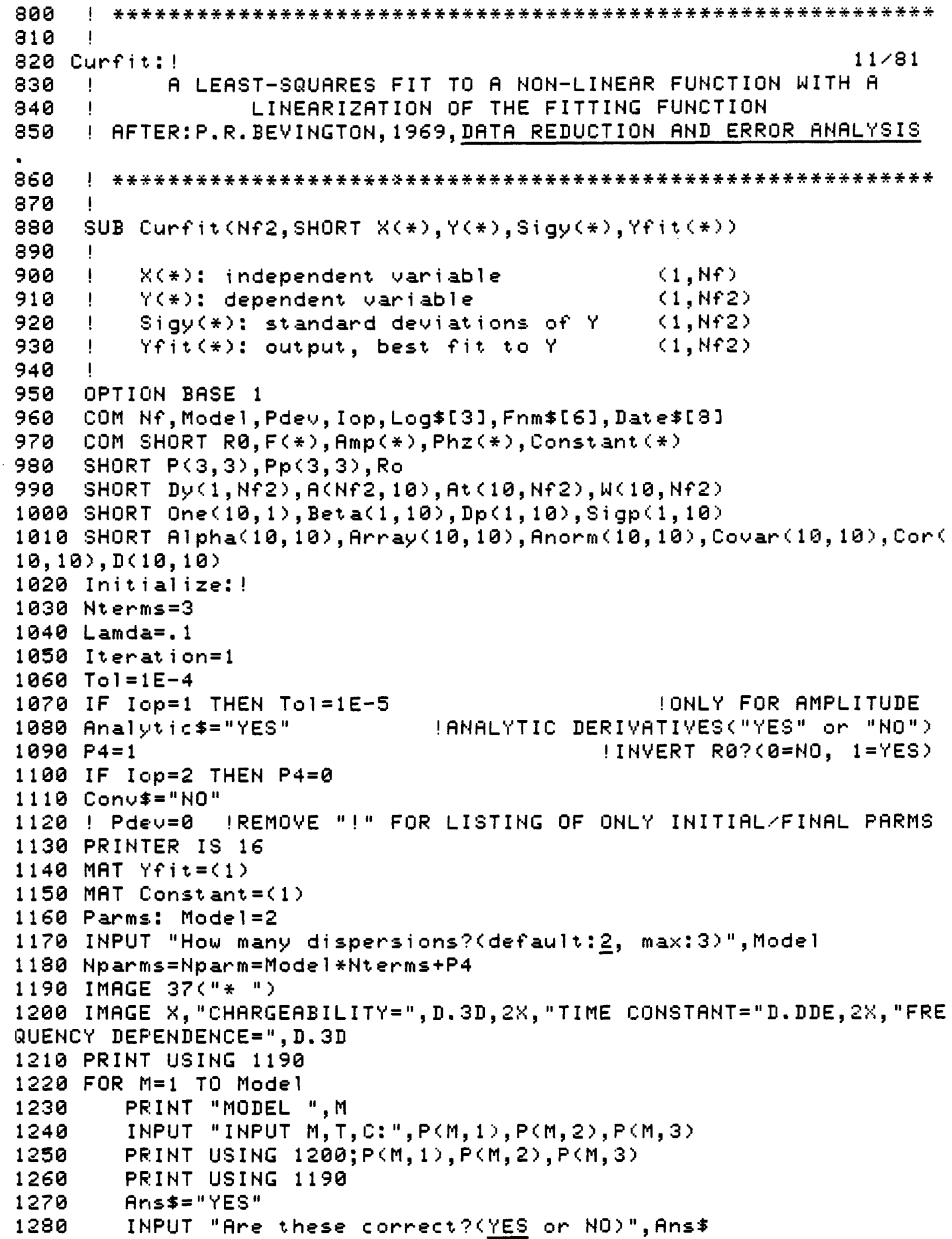




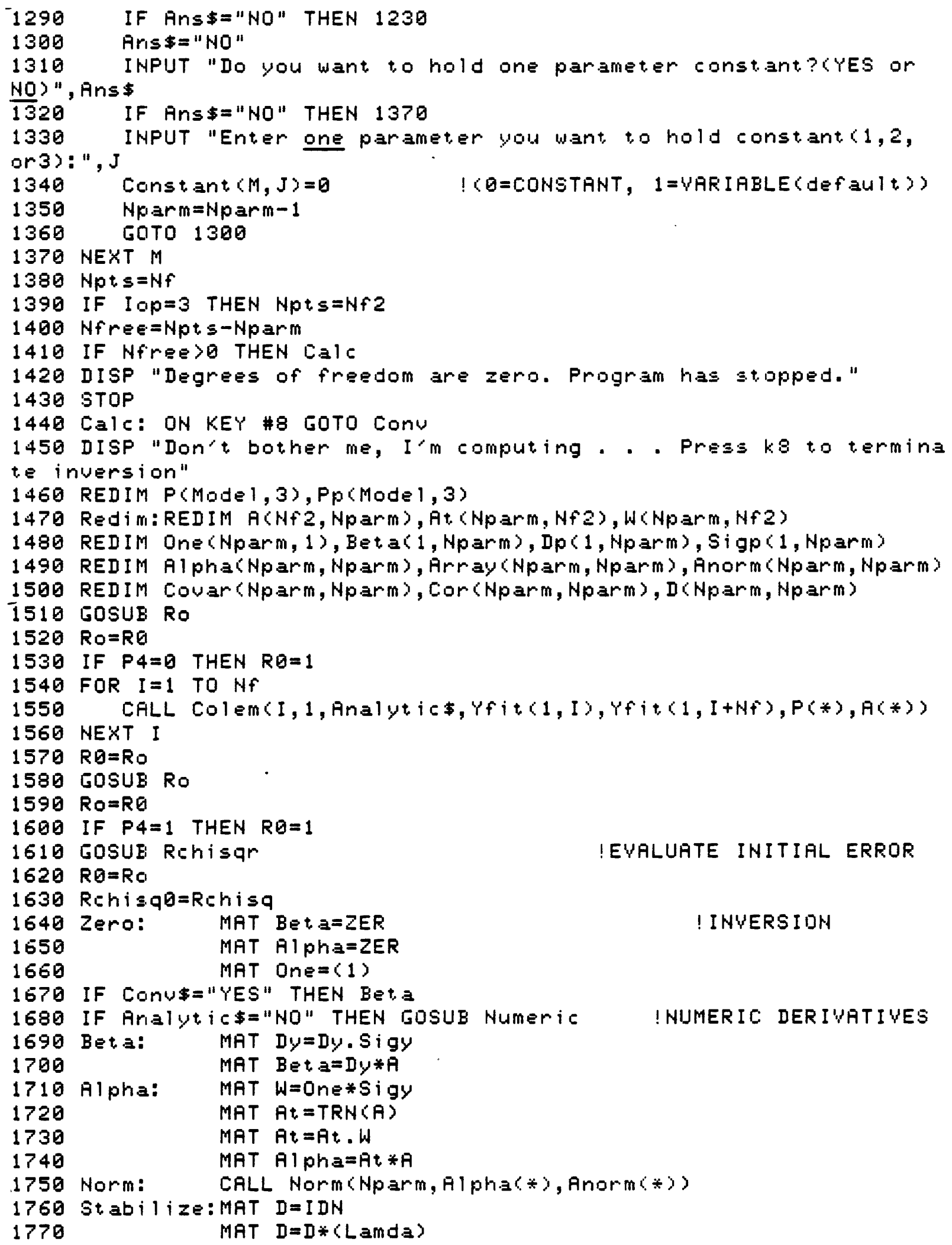




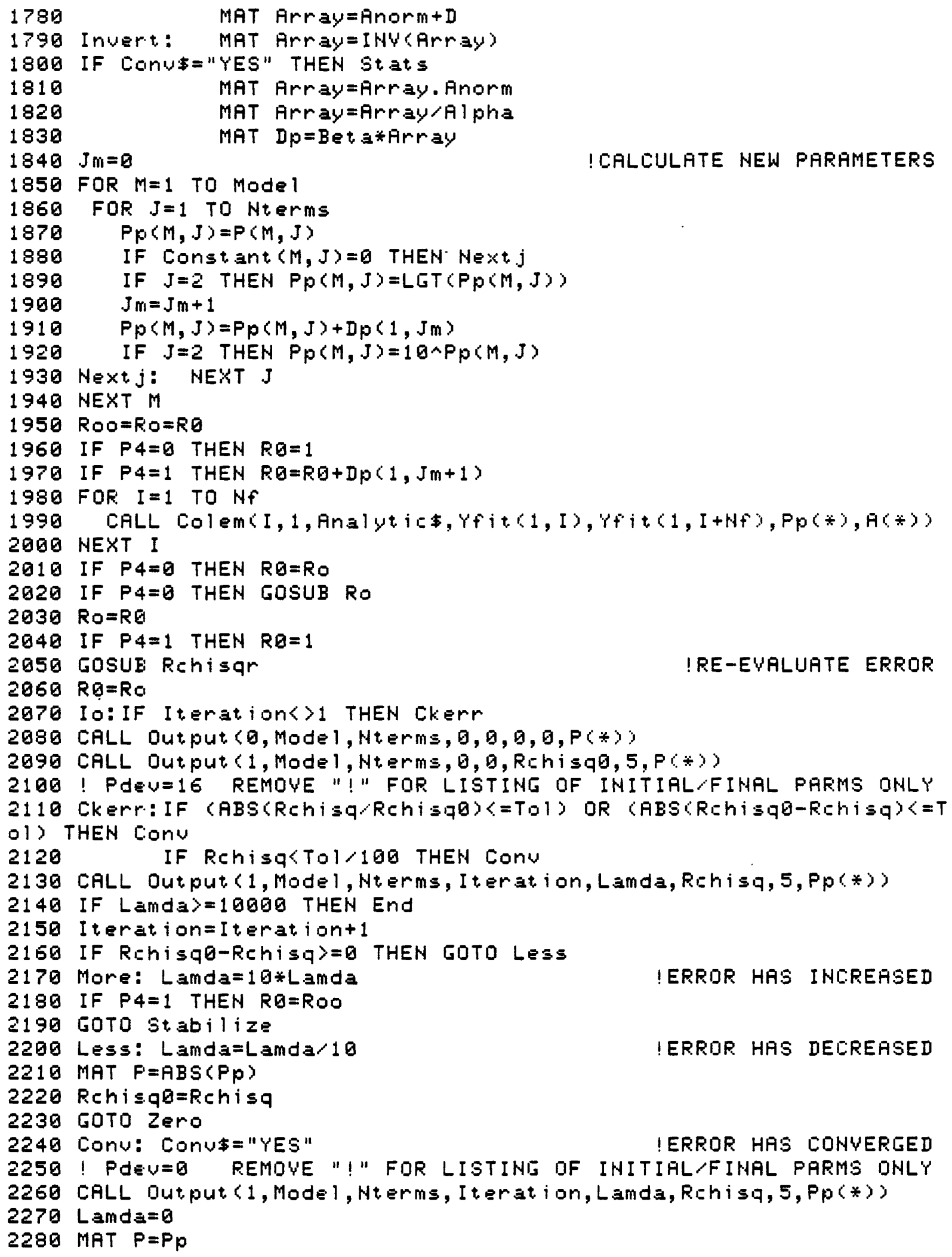




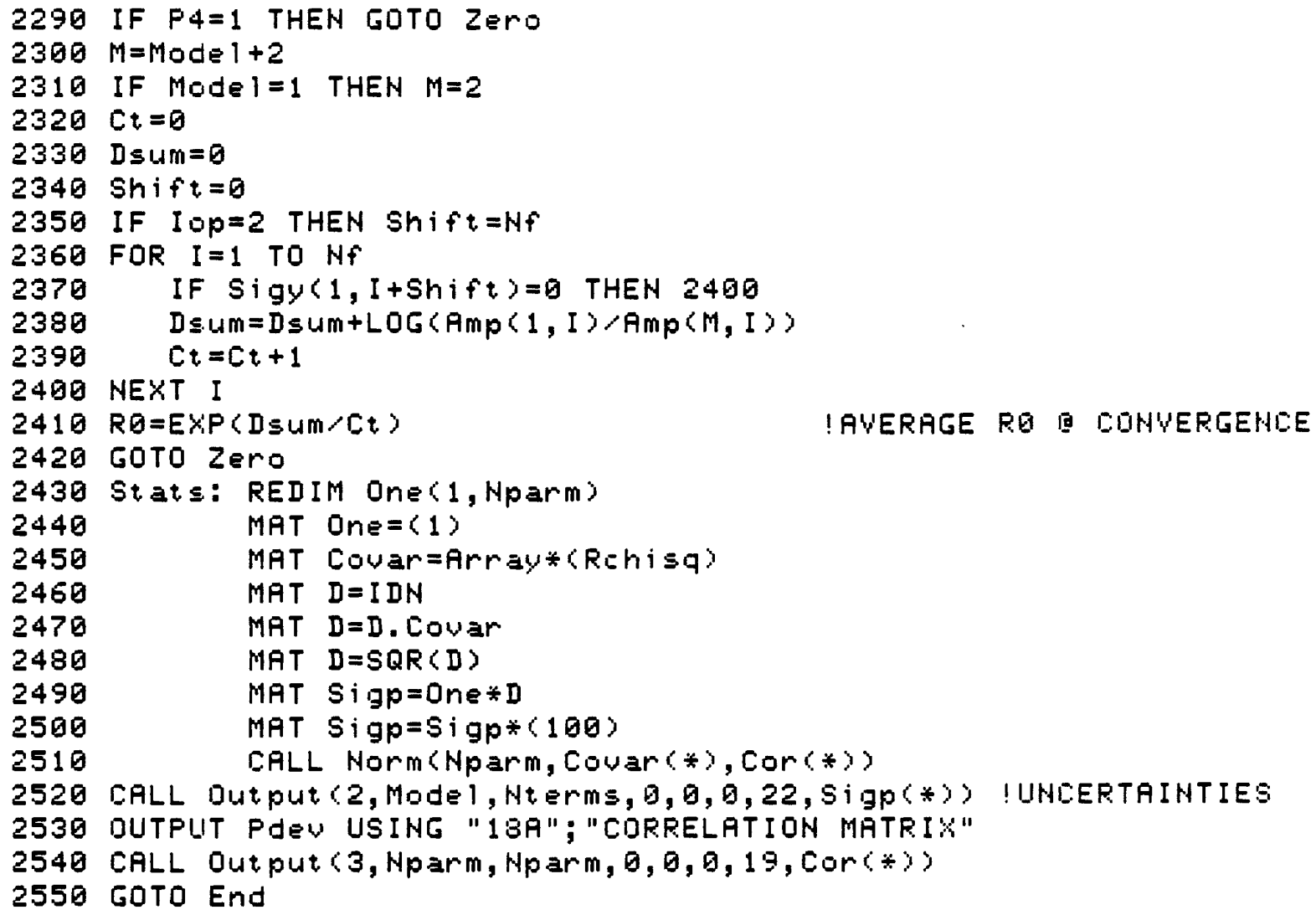




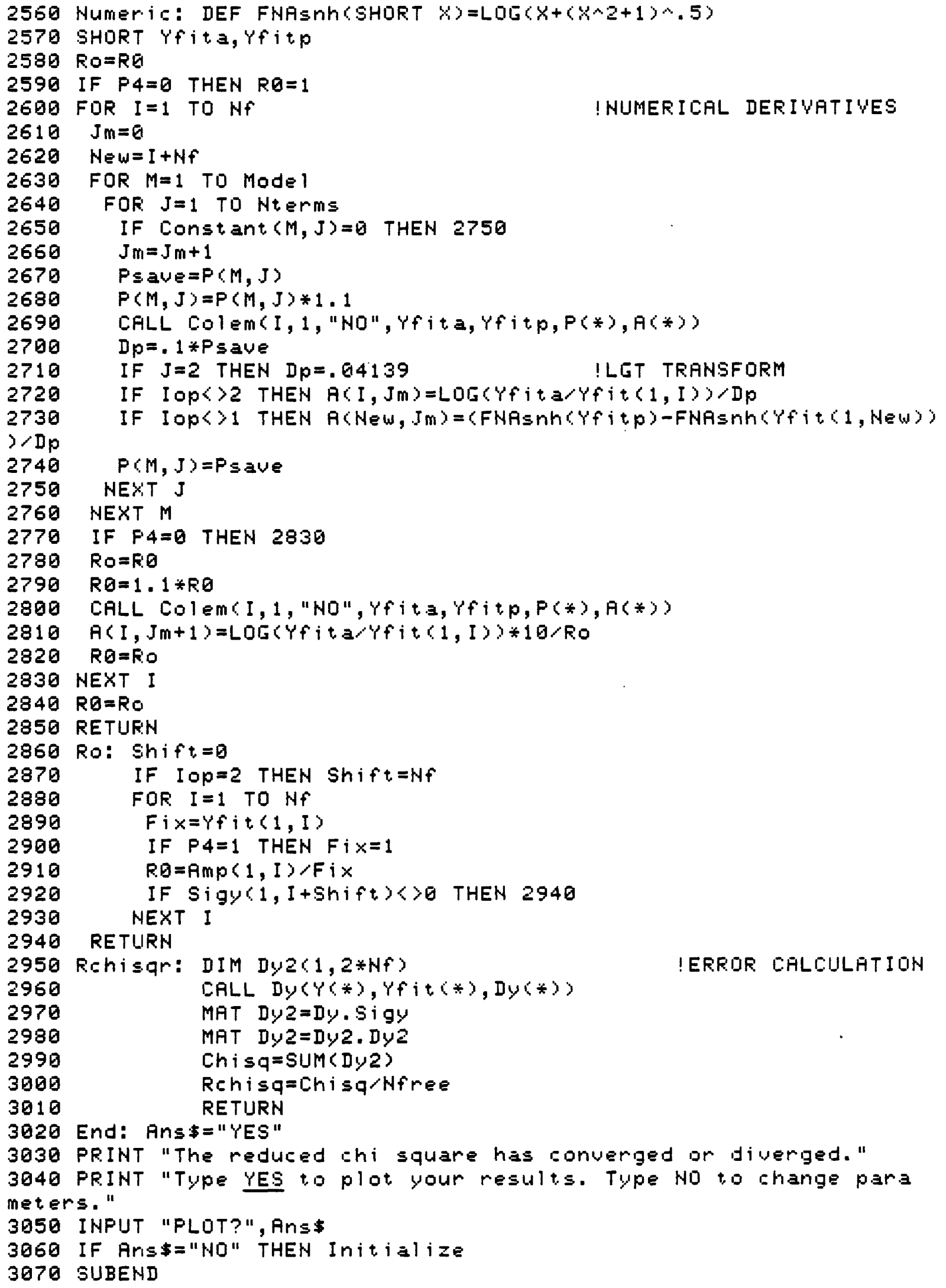




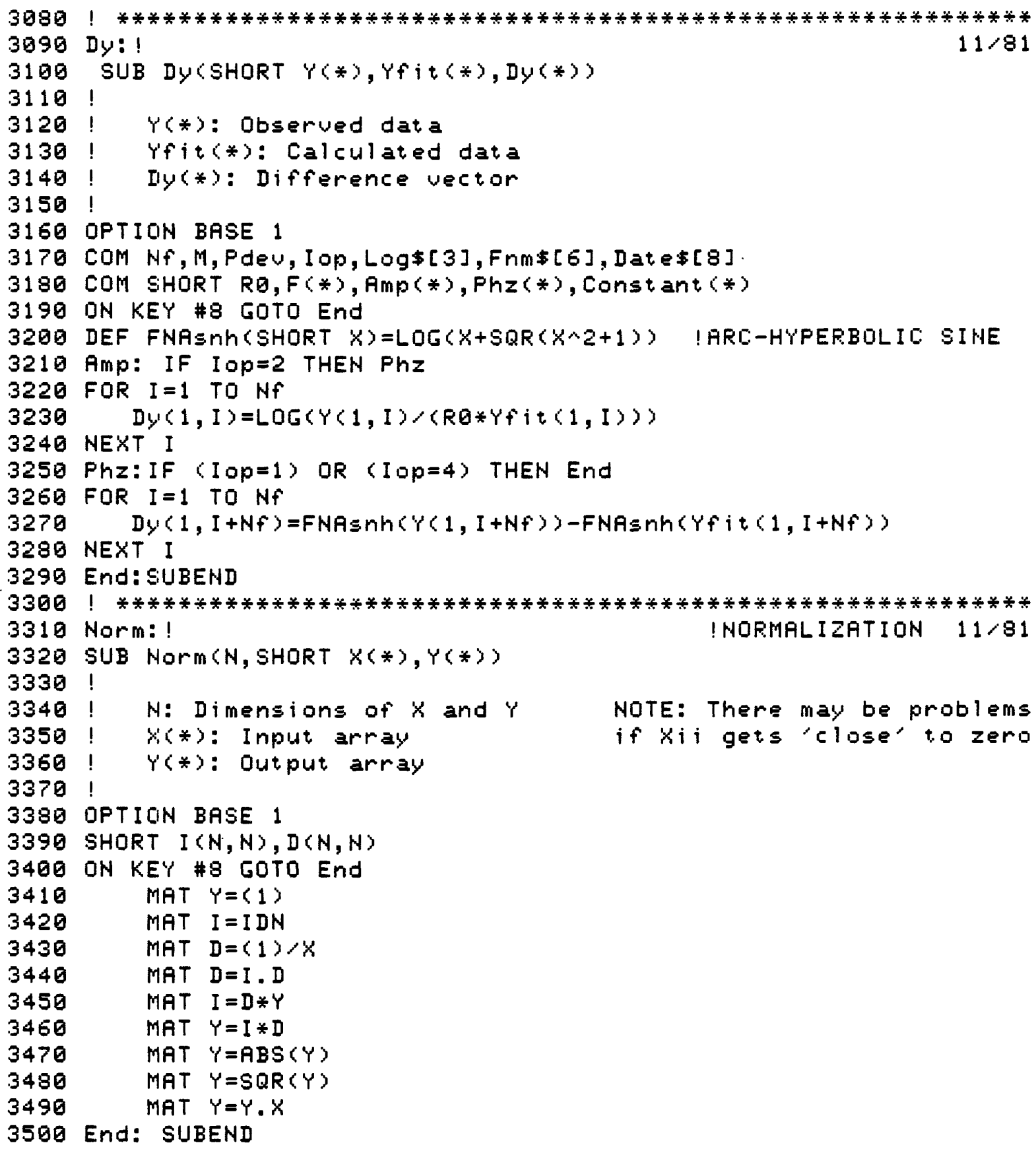




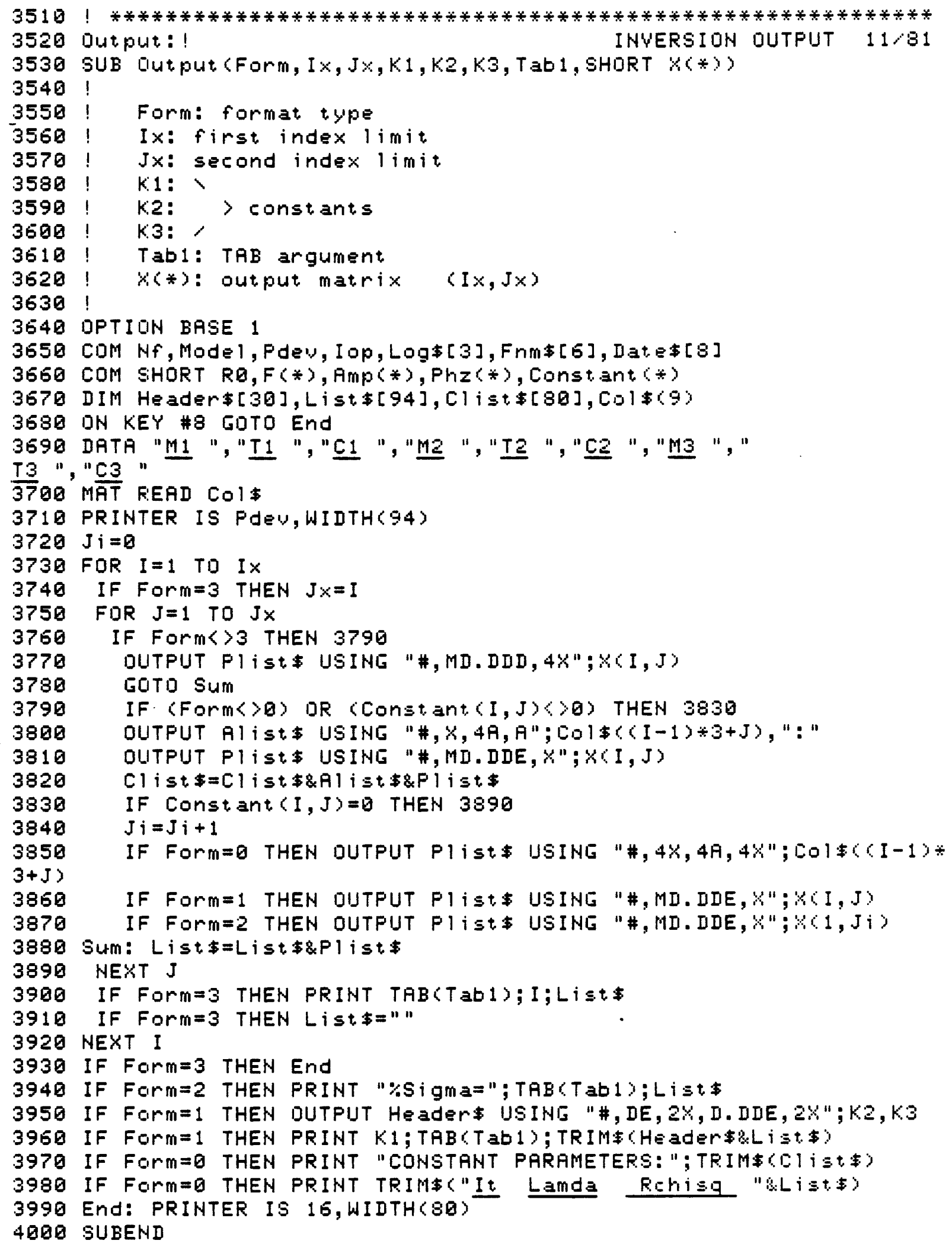




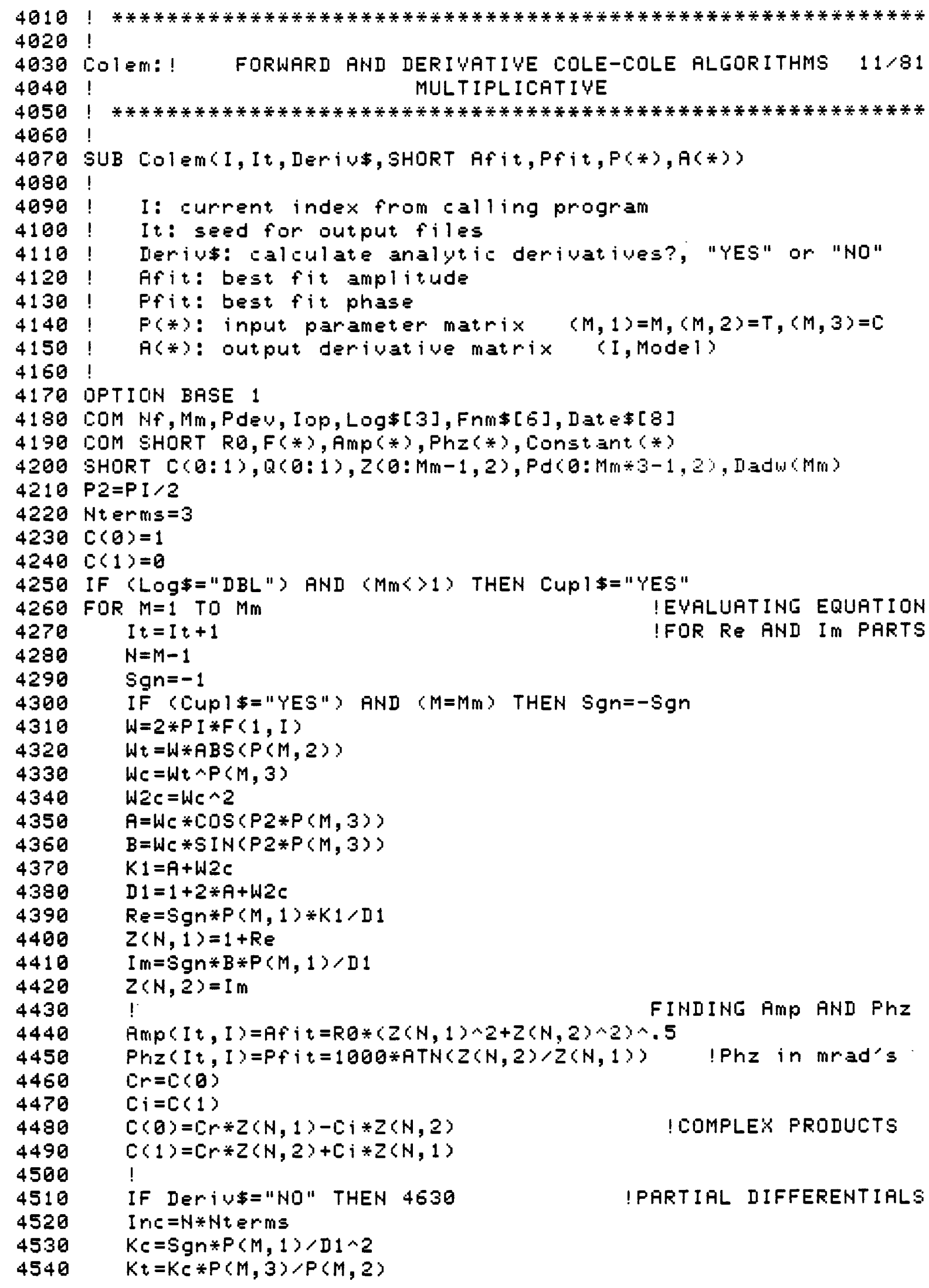




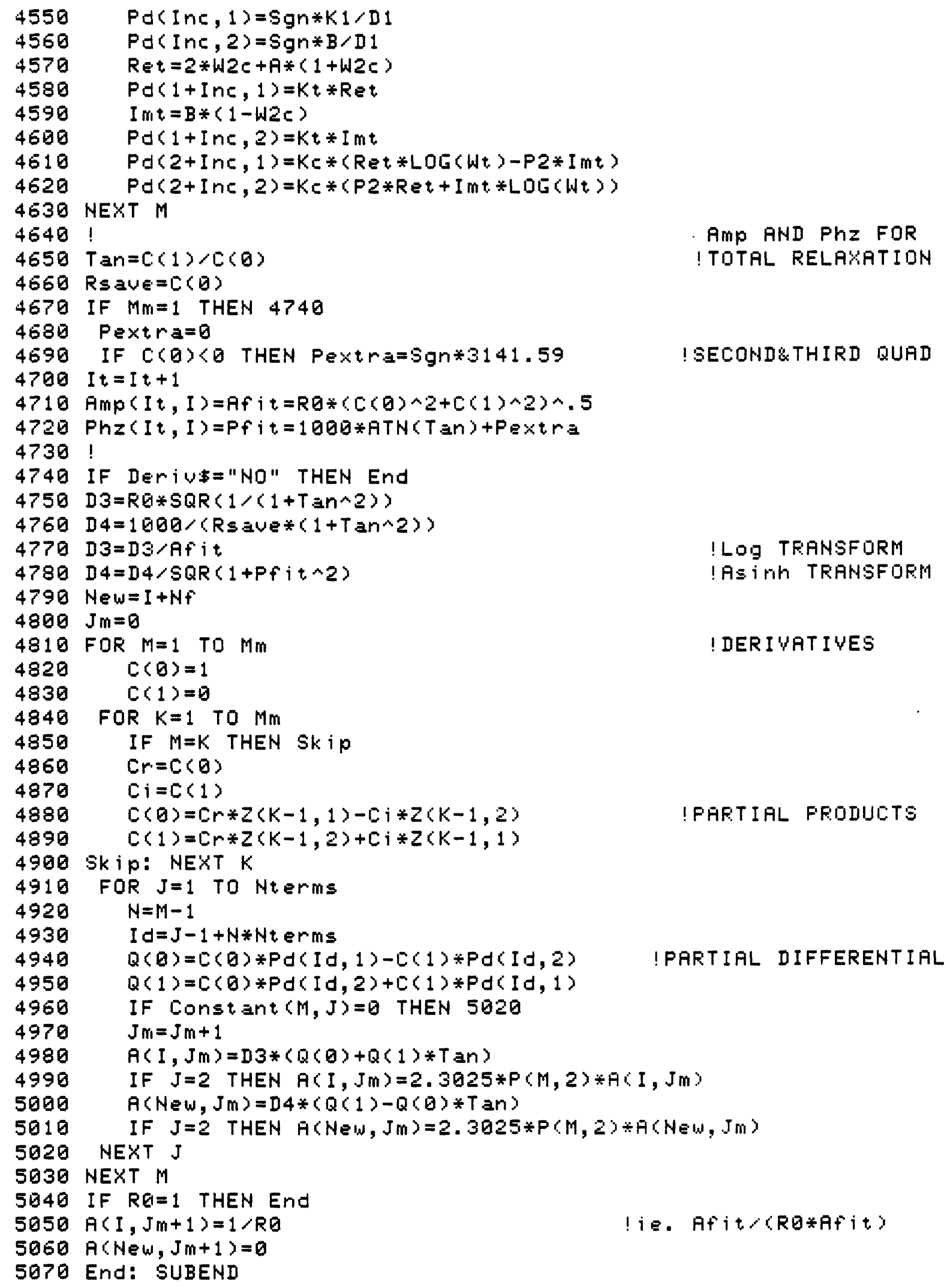




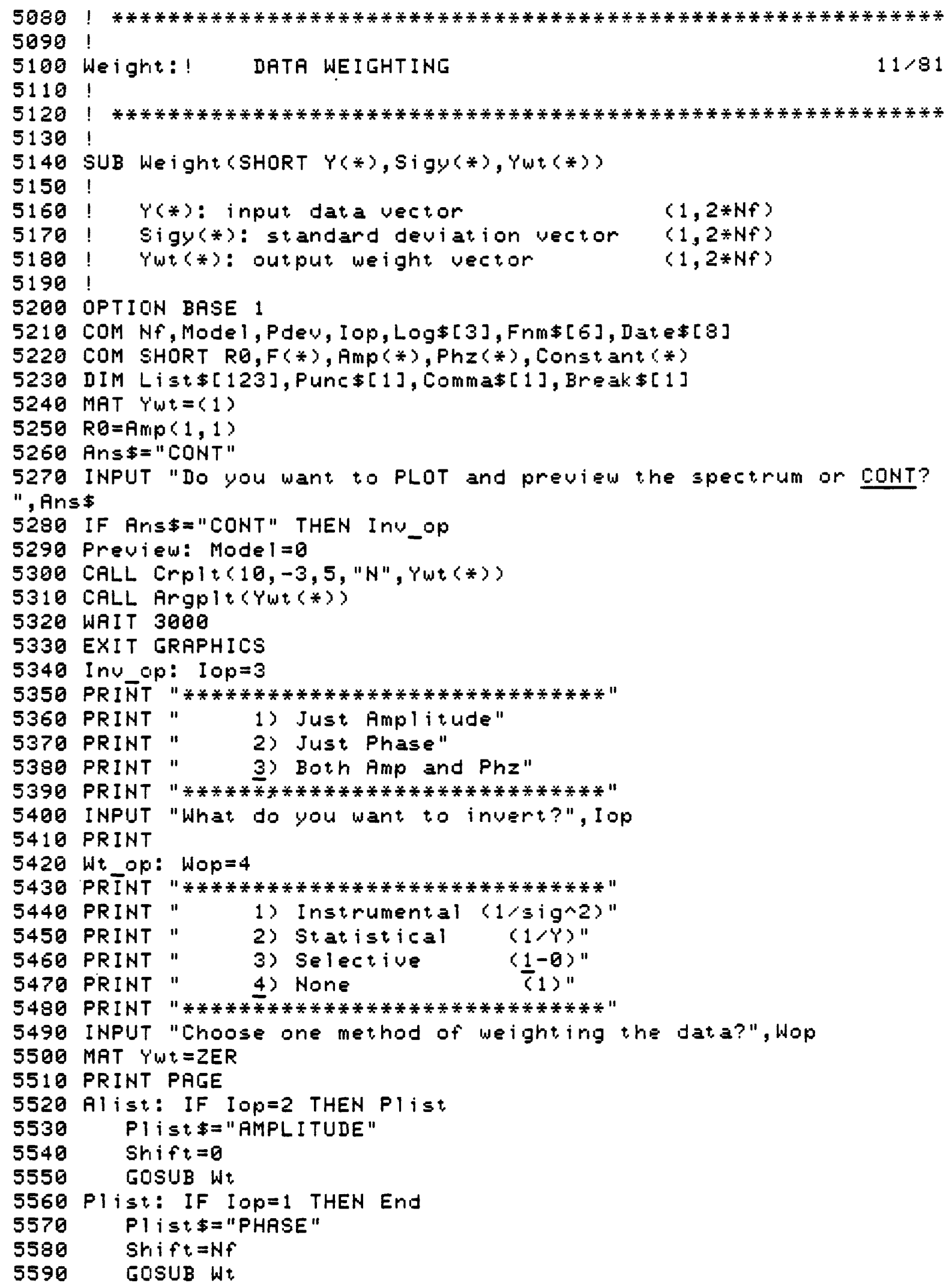




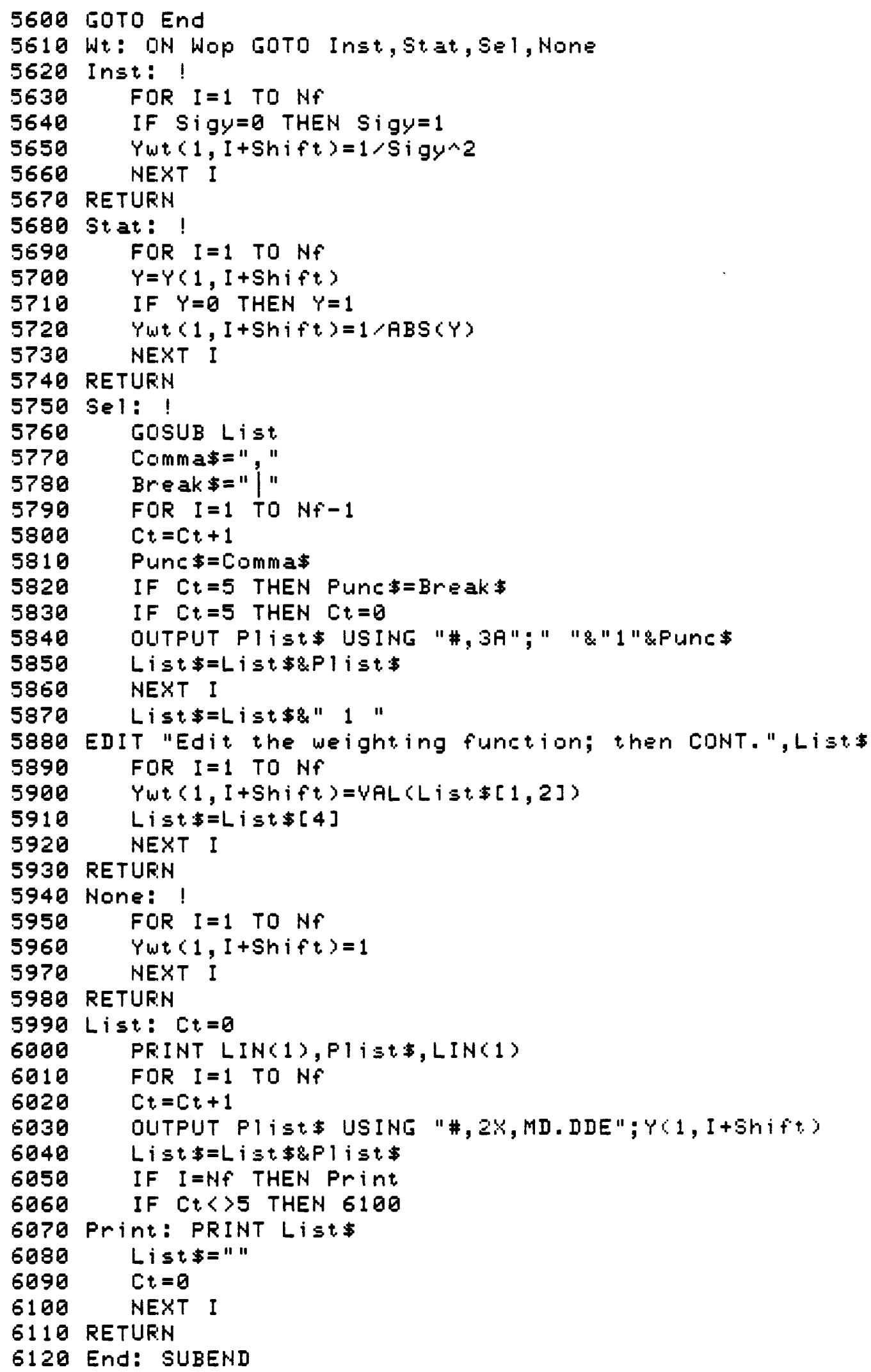




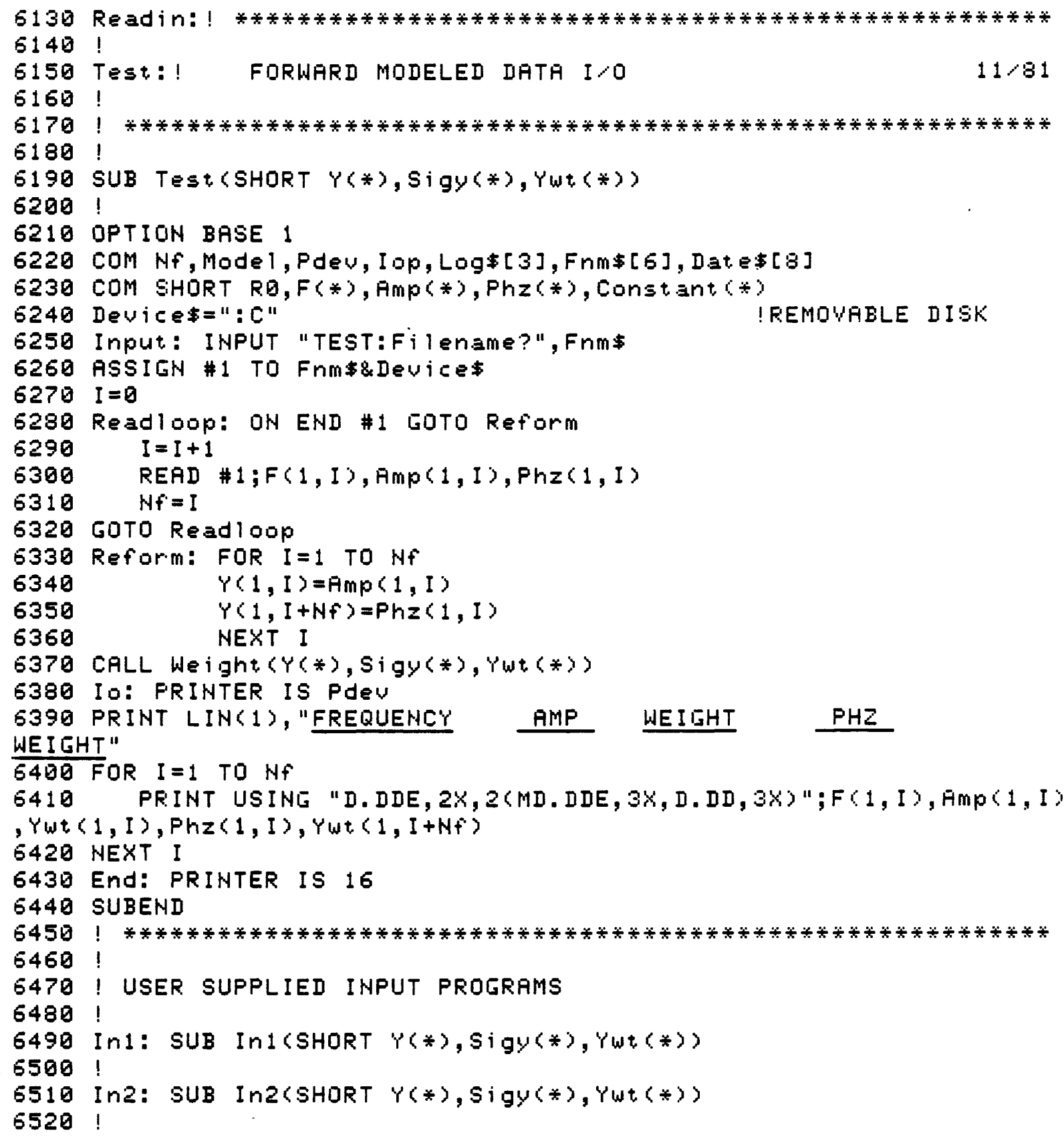




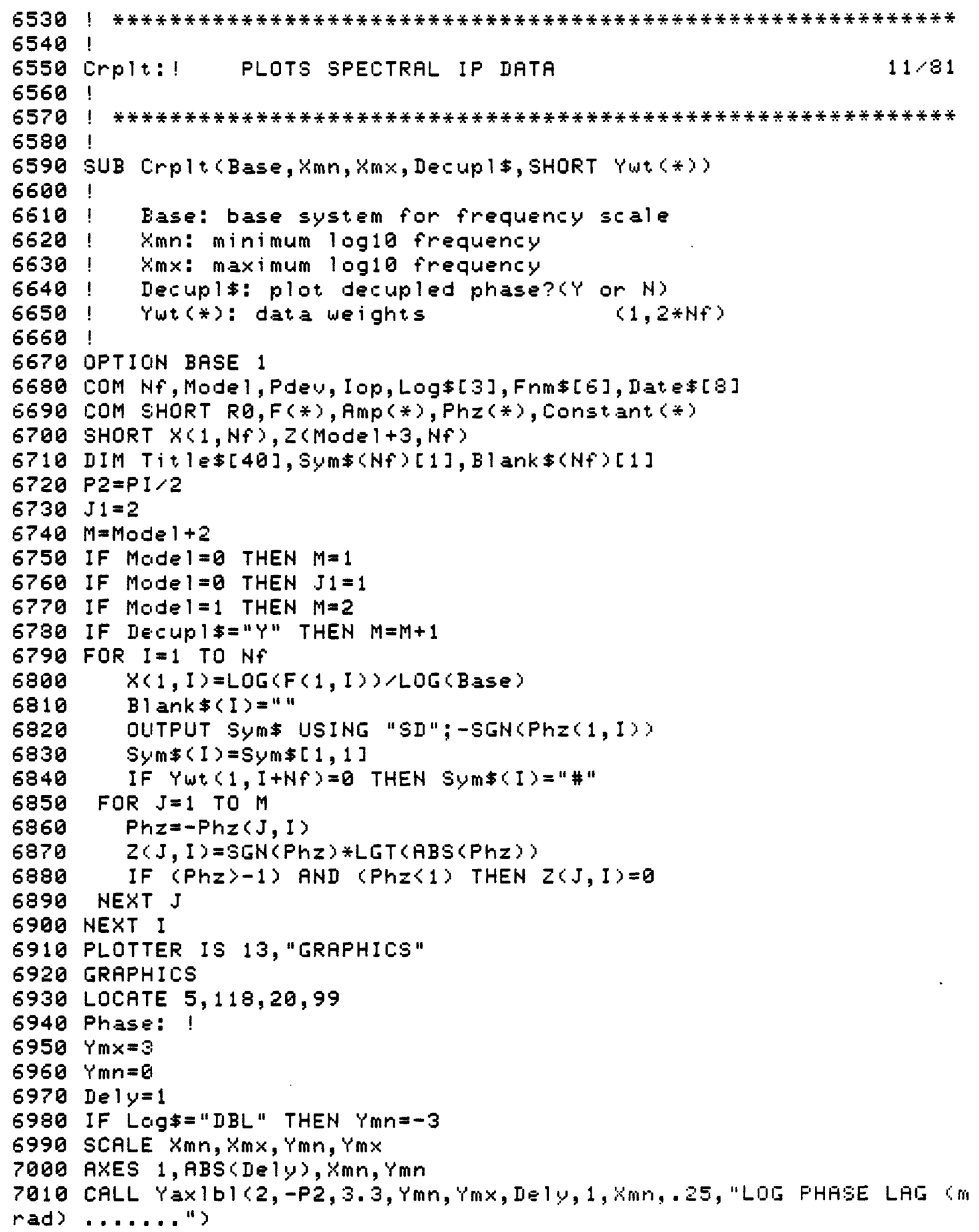




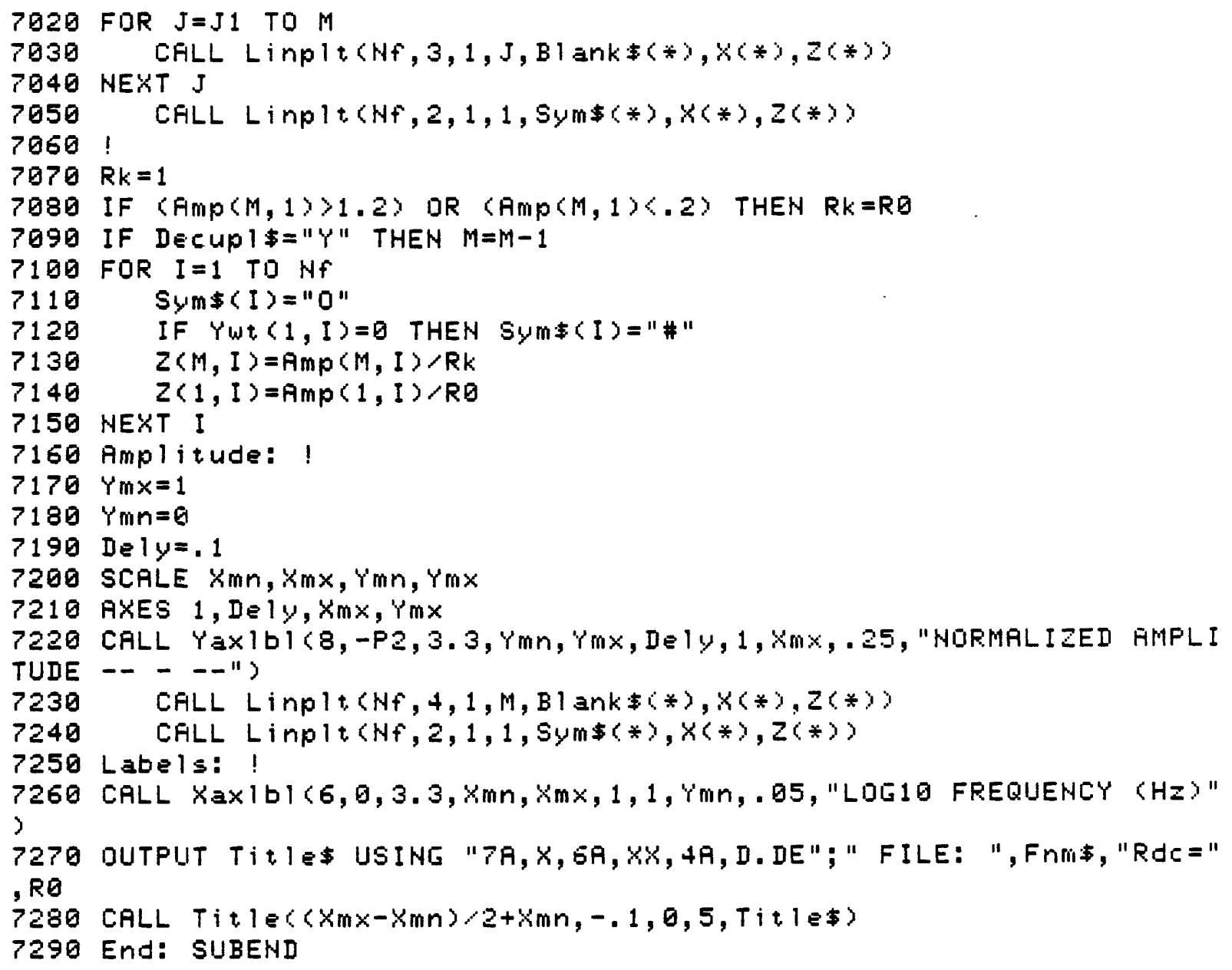




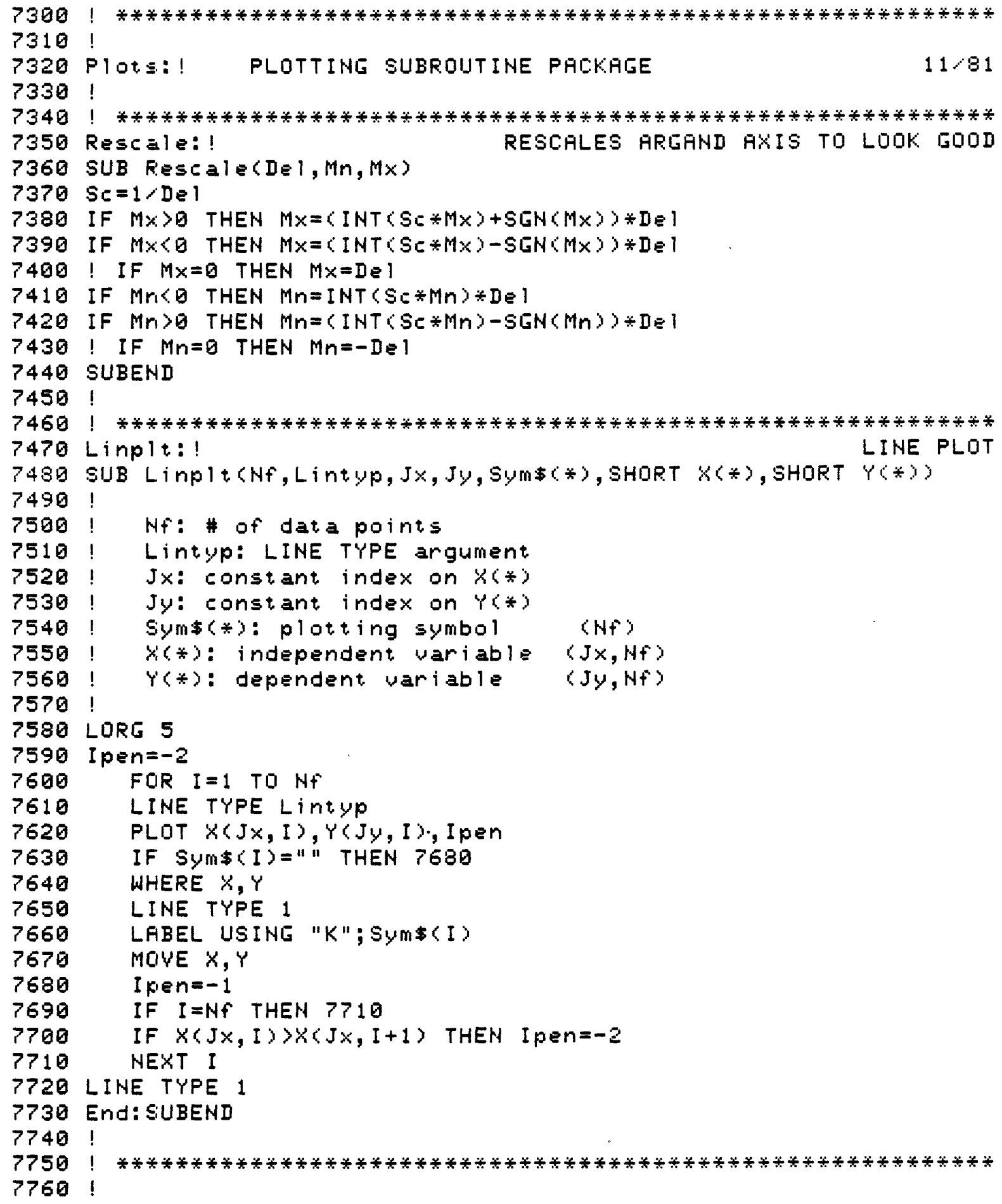




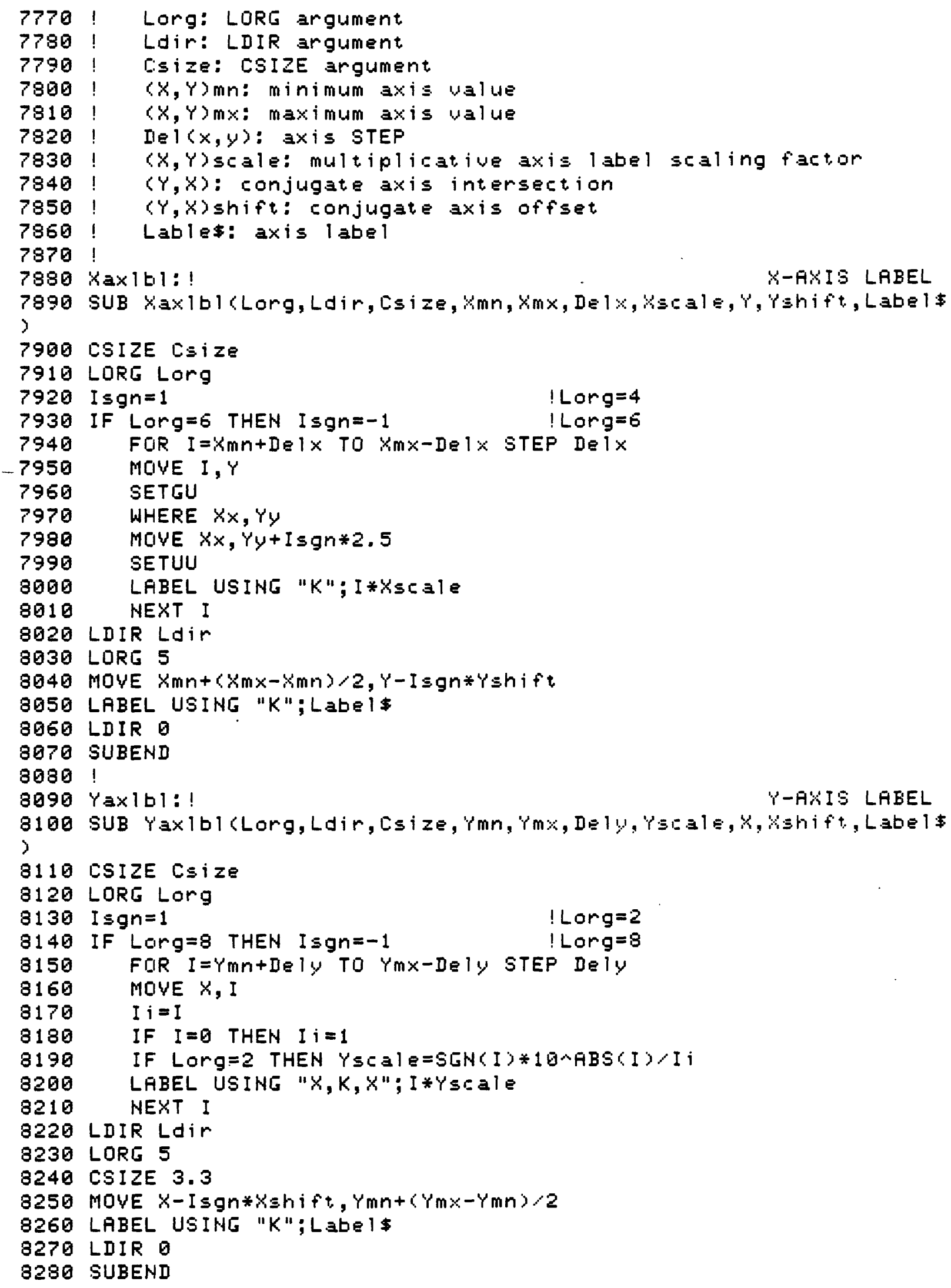




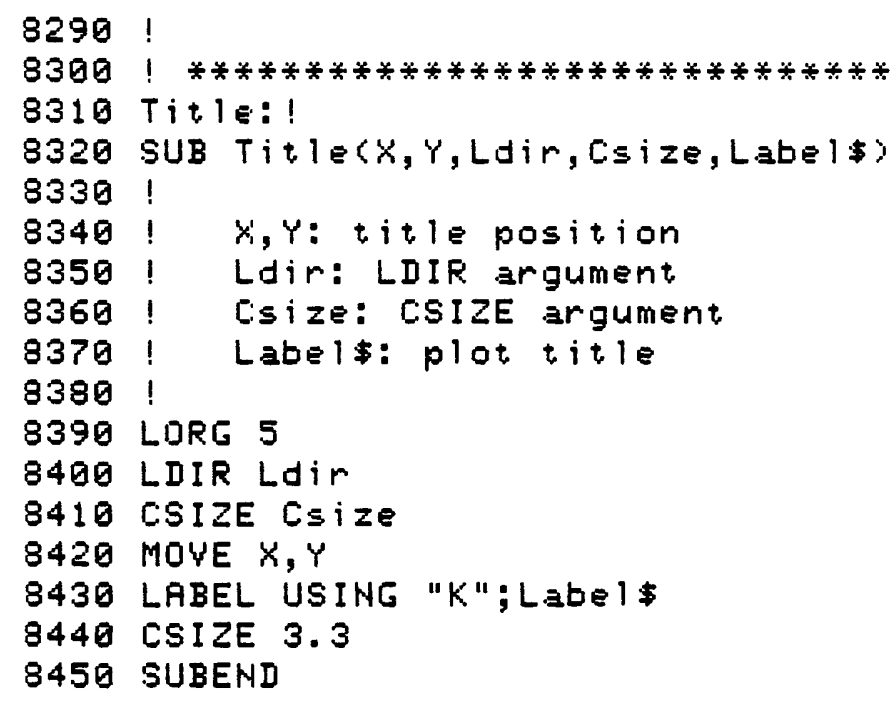




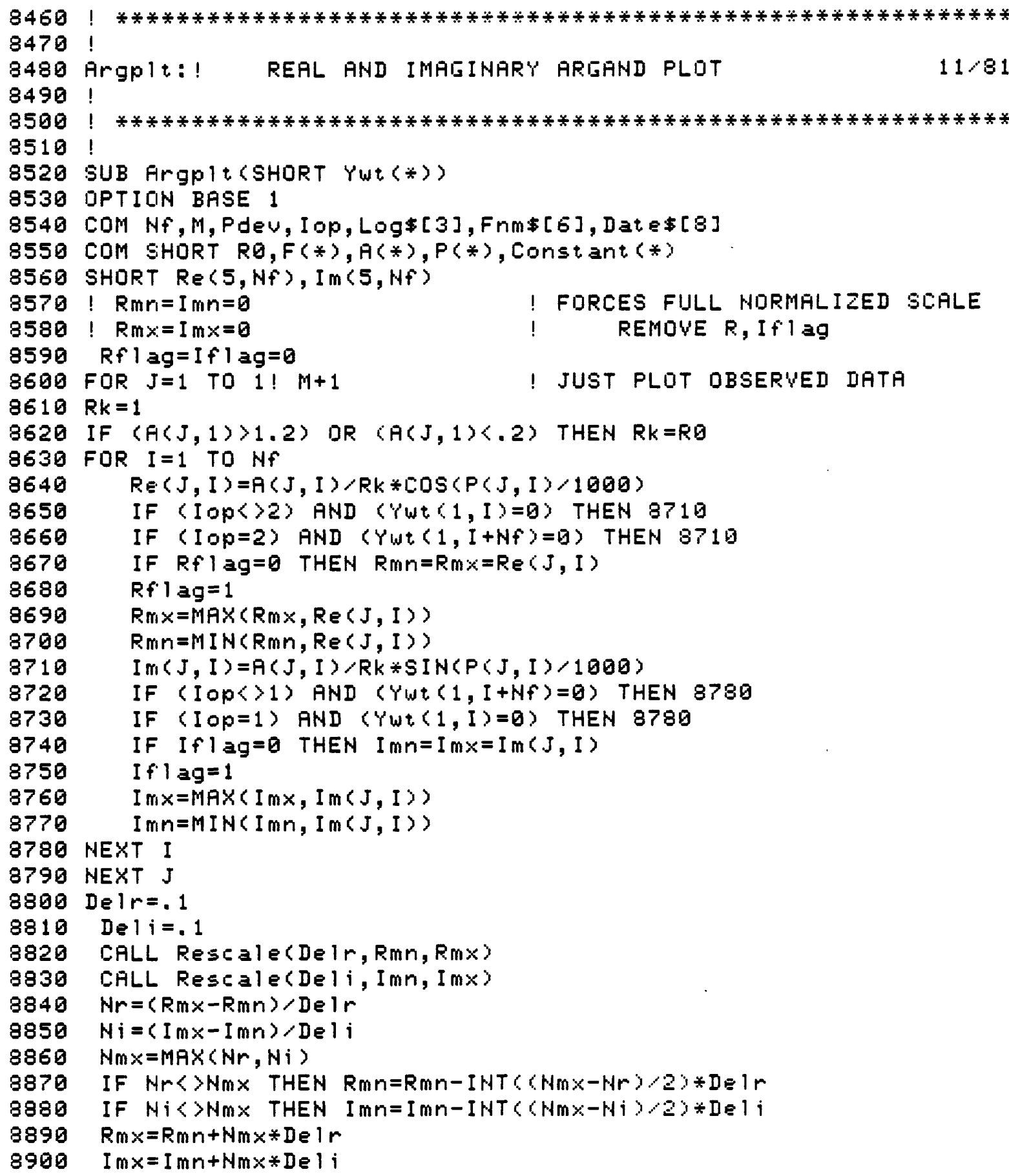




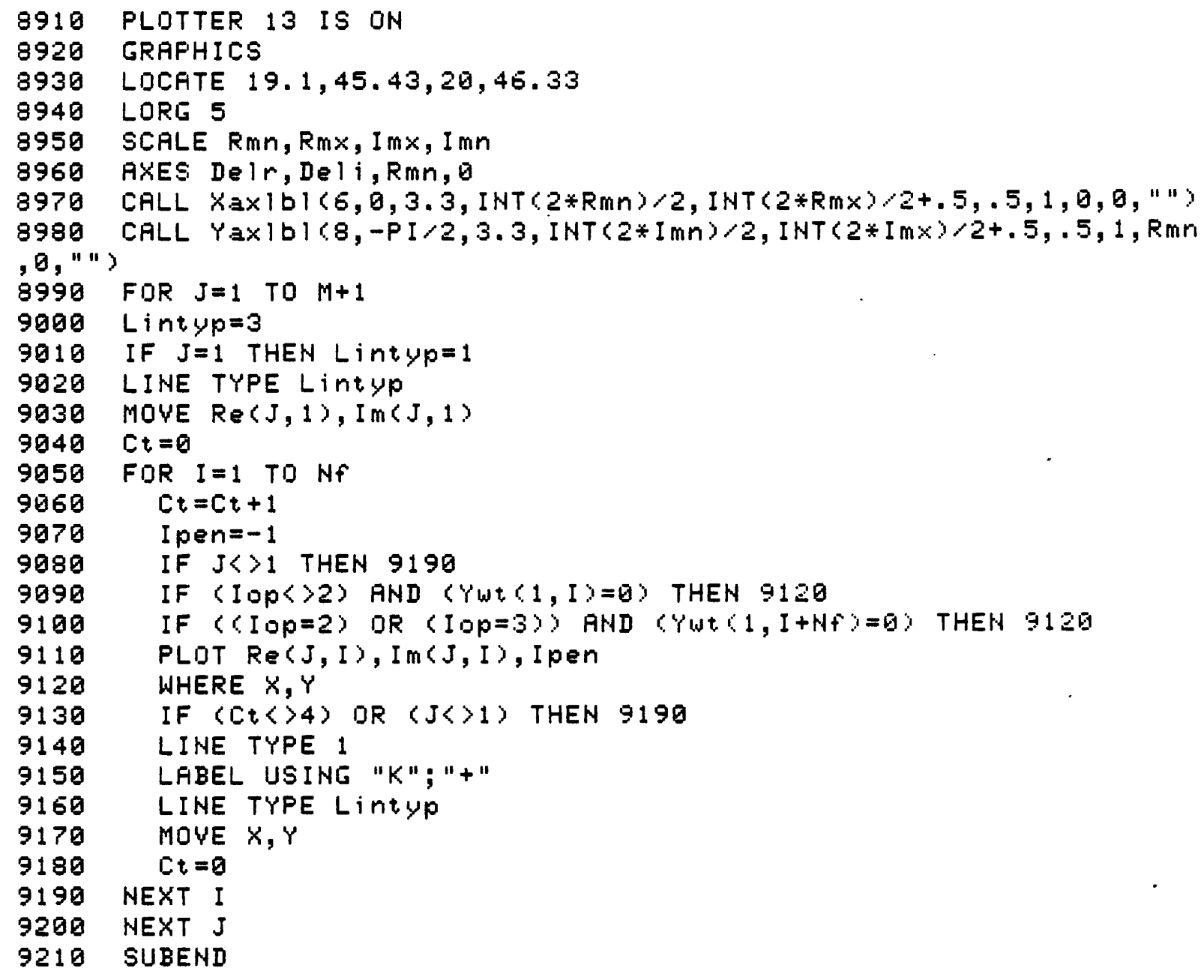

